\title{
The age of cataclysmic variables: a kinematical study
}

\author{
T. Ak ${ }^{\mathrm{a}, \mathrm{b}, *}, \mathrm{~S} . \mathrm{Bilir}^{\mathrm{a}}, \mathrm{S} . \mathrm{Ak}^{\mathrm{a}}$, K.B. Coşkunoğlu ${ }^{\mathrm{a}}$, Z. Eker ${ }^{\mathrm{b}, \mathrm{c}}$ \\ ${ }^{a}$ Istanbul University, Faculty of Sciences, Department of Astronomy and Space \\ Sciences, 34119 University, Istanbul, Turkey \\ b TÜBITTAK National Observatory, Akdeniz University Campus, 07058 Antalya, \\ Turkey \\ ${ }^{\mathrm{c}}$ Akdeniz University, Faculty of Arts and Sciences, Department of Physics, 07058, \\ Campus, Antalya, Turkey
}

\begin{abstract}
Using available astrometric and radial velocity data, the space velocities of cataclysmic variables (CVs) with respect to Sun were computed and kinematical properties of various sub-groups of CVs were investigated. Although observational errors of systemic velocities $(\gamma)$ are high, propagated errors are usually less than computed dispersions. According to the analysis of propagated uncertainties on the computed space velocities, available sample is refined by removing the systems with the largest propagated uncertainties so that the reliability of the space velocity dispersions was improved. Having a dispersion of $51 \pm 7 \mathrm{~km} \mathrm{~s}^{-1}$ for the space velocities, CVs in the current refined sample (159 systems) are found to have $5 \pm 1$ Gyr mean kinematical age. After removing magnetic systems from the sample, it is found that non-magnetic CVs (134 systems) have a mean kinematical age of $4 \pm 1$ Gyr. According to $5 \pm 1$ and $4 \pm 1$ Gyr kinematical ages implied by $52 \pm 8$ and $45 \pm 7$ $\mathrm{km} \mathrm{s}^{-1}$ dispersions for non-magnetic systems below and above the period gap, CVs below the period gap are older than systems above the gap, which is a result in agreement with the standard evolution theory of CVs. Age difference between the systems below and above the gap is smaller than that expected from the standard theory, indicating a similarity of the angular momentum loss time scales in systems with low-mass and high-mass secondary stars. Assuming an isotropic distribution, $\gamma$ velocity dispersions of non-magnetic CVs below and above the period gap are calculated $\sigma_{\gamma}=30 \pm 5 \mathrm{~km} \mathrm{~s}^{-1}$ and $\sigma_{\gamma}=26 \pm 4 \mathrm{~km} \mathrm{~s}^{-1}$. Small difference of $\gamma$ velocity dispersions between the systems below and above the gap may imply that magnetic braking does not operate in the detached phase, during which the system evolves from the post-common envelope orbit into contact.
\end{abstract}

Key words: 97.80.Gm Cataclysmic binaries, 98.10.+z Stellar dynamics and kinematics, 98.35.Pr Solar neighbourhood 


\section{Introduction}

Cataclysmic variables (hereafter CVs) are short-period interacting binary stars consisting of a white dwarf primary and low-mass secondary which overflows its Roche lobe and transfers matter to the white dwarf usually via a gas stream and an accretion disc. A bright spot is formed in the location where the matter stream impacts on the accretion disc. However disc formation is prevented in systems with strongly magnetic primaries. Nevertheless mass transfer continues through channels as accretion flows. For comprehensive reviews of the properties of CVs, see Warner (1995) and Hellier (2001).

According to standard CV formation and evolution scenario, CVs are formed from much wider -detached- main-sequence binaries with orbital periods typically 10-1000 d of the pairs with large mass ratios; secondaries being less than a solar mass, but the primaries are more massive $\left(M_{1} \simeq 1-9 M_{\odot}\right)$. The primary star evolves in a nuclear time scale of roughly $t_{n u c} \simeq 10^{10}\left(M_{1} / M_{\odot}\right)^{-3}$ yr (Kolb \& Stehle, 1996; Hellier, 2001). When the primary fills its Roche lobe, dynamically unstable mass transfer leads to a common envelope (CE) phase during which dynamical friction extracts orbital angular momentum to eject the envelope of the giant. The time spent in common envelope phase is very short compared to other phases of the evolution, of the order of $10^{3}-10^{4} \mathrm{yr}$. After the CE phase, system finds itself in a new detached phase called post common envelope phase or pre-CV state; the secondary component is still a main-sequence star, while the primary component has been changed to a white dwarf. This detached system approaches a semi-detached state by either the nuclear expansion of the secondary, or by shrinking orbit due to the loss of orbital angular momentum by gravitational radiation and magnetic braking. During the post-CE phase, the orbit shrinks from the post-CE period of about 0.1-10 d to the orbital period $P \simeq 9\left(M_{2} / M_{\odot}\right)$ h at the onset of mass transfer as a CV. The post-CE phase can be very short if the system emerges from the CE phase at almost semi-detached configuration (Kolb \& Stehle, 1996).

The standard scenario proposed for the evolution of CVs after the onset of the mass transfer is sensitive to orbital angular momentum loss mechanisms too as in the post CE phase of the evolution. It has been proposed two main mechanisms: magnetic braking and gravitational radiation. For CVs with orbital periods $(P)$ above the period gap, the secondary star is partially convective and, thus, it has a magnetic field. It has been proposed that the magnetic field in the secondary star causes orbital angular momentum loss by magnetic braking (Verbunt \& Zwaan, 1981; Rappaport et al., 1982, 1983;

\footnotetext{
* corresponding author.

Email address: tanselak@istanbul.edu.tr or

tanselak@tug.tubitak.gov.tr (T. Ak).
} 
Paczynski \& Sienkiewicz, 1983; Spruit \& Ritter, 1983; King, 1988) until the orbital period decreases to about $3 \mathrm{~h}$ at which the secondary star becomes fully convective and magnetic braking ceases. Consequently, the secondary star relaxes to its thermal equilibrium and shrinks inside its Roche lobe. At this point, the mass transfer shuts off completely and the gravitational radiation becomes dominant mechanism for the angular momentum loss (Paczynski, 1967). Since the mass transfer ceased, the system cannot be observed as a cataclysmic variable and it evolves towards shorter orbital periods through emission of gravitational radiation. At the orbital period of about $2 \mathrm{~h}$, the secondary star starts to fill its Roche lobe again and re-starts the mass transfer at a much lower rate than in the mode of long-period CVs. When the orbital period decreases to the observed minimum of about $80 \mathrm{~min}$, the secondary star becomes a degenerate (brown-dwarf like) object and, thus, further mass transfer causes the orbital period to increase (Patterson, 1998), creating systems called period bouncers. For a comprehensive review of the formation and evolution of CVs, see Ritter (2008).

Though the above model successfully explains the period gap, i.e. drop in the number of known CVs in between orbital periods in the range about $2<P(\mathrm{~h})<3$, some predictions of it are contradictory with observations. Since CVs should spend most of their lifetime near the period minimum, a significant accumulation of CVs near the period minimum is expected. Although such an accumulation has not been observed for many years (Kolb \& Baraffe, 1999), there is now a claimed discovery of a spike at the period minimum (Southworth et al., 2008; Gänsicke et al., 2009). According to population synthesis (de Kool, 1992; de Kool \& Ritter, 1993; Kolb, 1993; Politano, 1996; Kolb \& Baraffe, 1999; Howell et al., 1997; King \& Schenker, 2002), the vast majority of CV population should have orbital periods shorter than about $2 \mathrm{~h}$. However, such an accumulation has not been seen in the distribution of orbital periods of CVs. Recently, Littlefair et al. (2008) found seven systems with brown dwarf donors and proposed that the missing population of post-period minimum CVs has finally been identified, although their masses and radii are inconsistent with model predictions. In addition, the predicted space density of CVs is about a few $10^{-5}$ to a few $10^{-4} \mathrm{pc}^{-3}$ (de Kool, 1992; Politano, 1996), whereas the space density derived from the observations is in agreement only with the lower limit of these predictions (Pretorius et al., 2007a; Schwope et al., 2002; Ak et al., 2008). Although a number of alternative suggestions (Livio \& Pringle, 1994; King \& Kolb, 1995; Clemens et al., 1998; Kolb et al., 1998) and some alternative angular momentum loss mechanisms (Andronov et al., 2003; Taam \& Spruit, 2001; Schenker et al., 2002; Willems et al., 2005, 2007) has been introduced as possible solutions to these problems, none of them could solve the problem with a complete success.

The observational data sets, which can be used to test the above predictions of the standard evolution model of CVs, are strongly biased by the selection ef- 
fects. Brightness dependent selection effects are the strongest since the known CV sample is not approximately magnitude-limited, even if a limiting magnitude as bright as $V=13$ is adopted (Pretorius et al., 2007b). Nevertheless, kinematical age of a cataclysmic variable, which is a time span since formation of component stars, can be used to test the predictions of the model. The age of a CV does not affect its mass transfer rate at a given orbital period. Hence the age distribution of CVs is not biased by brightness-selection (Kolb, 2001). Nevertheless, shorter the orbital period, it is harder to measure radial velocity variation for determining orbital parameters.

Kolb \& Stehle (1996), who determined the age structure of a modeled population for galactic CVs by applying standard models for the formation and evolution of $\mathrm{CVs}$, predicted that systems above the period gap ( $P \gtrsim 3 \mathrm{~h}$ ) must have an average age of $1 \mathrm{Gyr}$, with most of them being younger than $1.5 \mathrm{Gyr}$, while systems below the gap $(P \lesssim 2 \mathrm{~h}$ ) should display a wide range of all ages above about 1 Gyr, with a mean of 3-4 Gyr (see also Ritter \& Burkert, 1986). Using the empirical relation between age $t$ and space velocity dispersion $\sigma(t)$ of field stars in the solar neighbourhood (Wielen, 1977; Wielen et al., 1992), $\sigma(t)=k_{1}+k_{2} t^{1 / 2}\left(k_{1}\right.$ and $k_{2}$ are constants), an intrinsic dispersion of the $\gamma$ velocities $\sigma_{\gamma} \simeq 15 \mathrm{~km} \mathrm{~s}^{-1}$ for the systems above the period gap, and $\sigma_{\gamma} \simeq 30$ $\mathrm{km} \mathrm{s}^{-1}$ below the gap were predicted by Kolb \& Stehle (1996). Kolb (2001) states that the difference between ages of systems above and below the period gap is mainly due to the time spent evolving from the post-common envelope (post-CE) orbit into contact. However, van Paradijs et al. (1996) (hereafter referred to as vPAS96), who collected the observed $\gamma$ velocities for a sample of CVs from published radial velocity studies and analysed this CV sample statistically, could not detect such a difference between the velocity dispersions for the systems above and below the period gap. Using precise $\gamma$ velocity measurements of only four dwarf novae above the period gap, North et al. (2002) suggest a velocity dispersion of $\sim 8 \mathrm{~km} \mathrm{~s}^{-1}$. It should be noted that Kolb (2001) also expresses that if magnetic braking does not operate in the detached phase, the $\gamma$ velocity dispersions of CVs are $\sigma_{\gamma} \simeq 27 \mathrm{~km} \mathrm{~s}^{-1}$ above the period gap versus $\sigma_{\gamma} \simeq 32 \mathrm{~km} \mathrm{~s}^{-1}$ below the gap.

Aim of this paper is to derive the observed $\gamma$ velocity dispersions to test predictions. Kinematical age profiles for CVs according to different orbital period regimes are investigated in order to understand orbital period evolution of CV orbits. In order to do this, we have collected the measured $\gamma$ velocities of CVs from the published radial velocity studies since the work of vPAS96 and combined our $\gamma$ velocity collection with their sample. 
Table 1

The data sample. CV denotes CVs with unknown types, DN dwarf novae, NL novalike stars and $\mathrm{N}$ novae. The last column is for references. If there is only one number, it refers to colour excess. If there are two numbers, the first one is for colour excess and the other for orbital period. First five lines of Table 1 are given here. The table can be obtained electronically.

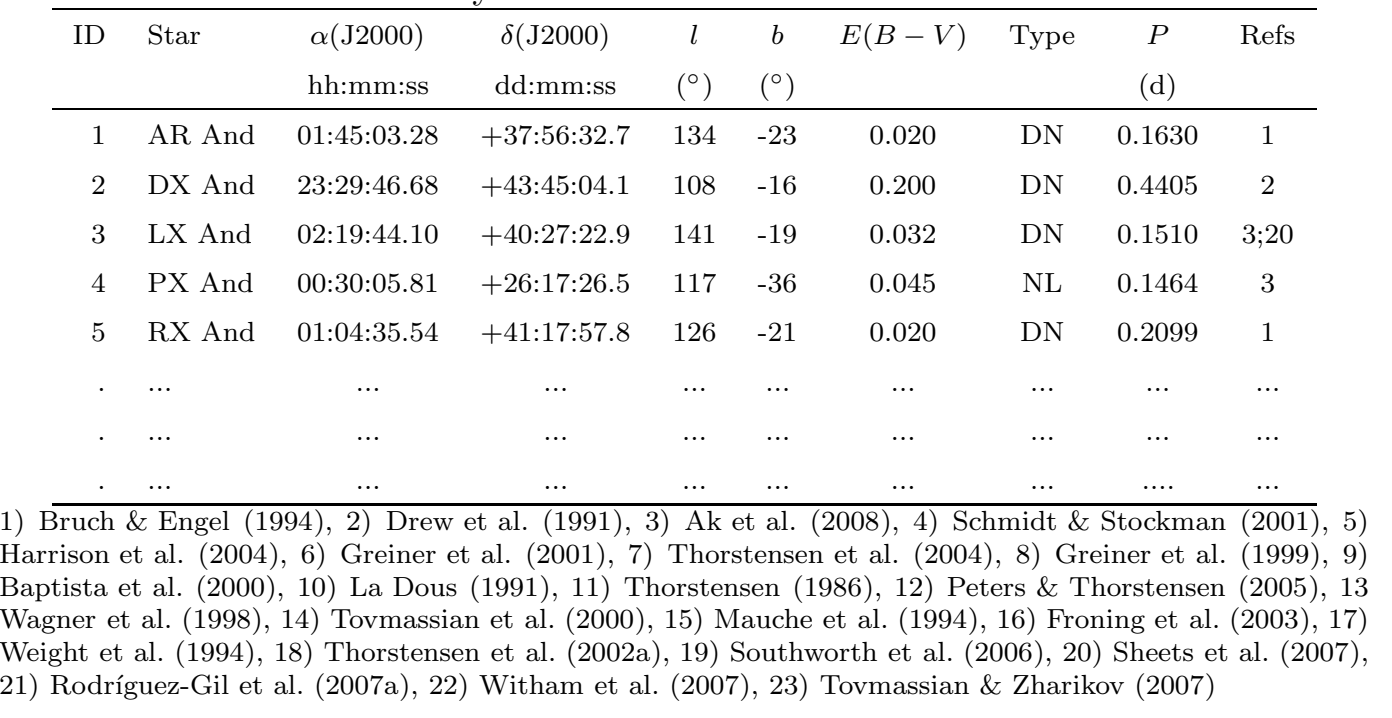

\section{The data}

Distances (or parallaxes), proper motions and $\gamma$ velocities were collected for 194 CVs which are listed in Table 1 . The columns of the table are organized as name, equatorial $(\alpha, \delta)$ and galactic $(l, b)$ coordinates, colour excess $E(B-V)$, type of the $\mathrm{CV}$, and its orbital period. The types, equatorial coordinates and orbital periods of CVs were mostly taken from Ritter \& Kolb (2003, Edition 7.7) and Downes et al. (2001).

\subsection{Distances and proper motions}

First precise trigonometric parallaxes of the brightest CVs were obtained from Hipparcos Satellite (Duerbeck, 1999). Trigonometric parallaxes of some CVs later were measured by using either Hubble Space Telescope's Fine Guidence Sensor (McArthur et al., 1999, 2001; Beuermann et al., 2003, 2004; Harrison et al., 2004; Roelofs et al., 2007) or the ground-based observations (Thorstensen, 2003; Thorstensen et al., 2008), as well. However, number of systems with distances obtained from the trigonometric parallax method is only about 30 . Nevertheless, there exist reliable distance prediction techniques for the systems whose trigonometric parallaxes do not exist. The distances were predicted using the period-luminosity-colours (PLCs) relation of Ak et al. (2007a). Using 2MASS $\left(J H K_{s}\right)$ photometric data, the PLCs relation of Ak et al. (2007a) 
Table 2

Star, parallaxes $(\pi)$, proper motions $\left(\mu_{\alpha} \cos \delta, \mu_{\delta}\right)$, systemic velocities $(\gamma)$ and space velocity components $(U, V, W)$ for the systems listed in Table 1 . In each row, the first and second references are for the parallax and the proper motion, respectively. The third and further references are for $\gamma$ velocity. First five lines of Table 2 are given here. The table can be obtained electronically.

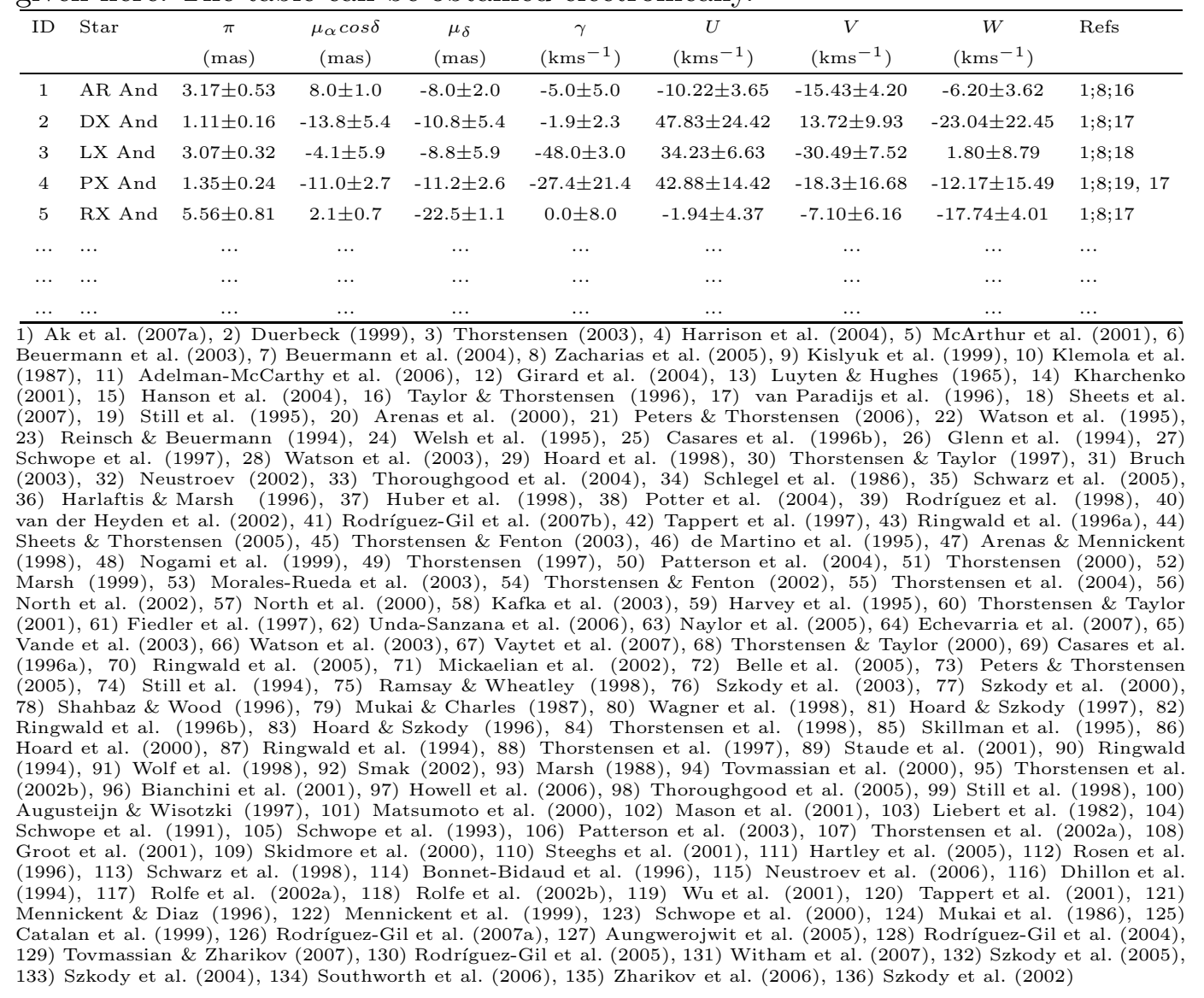

for $\mathrm{CVs}$ is reliable and valid in the ranges $0.032<P(d) \leq 0.454,-0.08<$ $(J-H)_{0} \leq 1.54,-0.03<\left(H-K_{s}\right)_{0} \leq 0.56$ and $2.0<M_{J}<11.7 \mathrm{mag}$, which are well covering the present data of this study. For a detailed description of the method by the PLCs relation, see Ak et al. (2007a, 2008).

The proper motions of CVs were mostly taken from the NOMAD Catalogue of Zacharias et al. (2005). Distribution of parallax errors and proper motion errors of the present CV sample are shown in Fig. 1a-b, respectively. The median value and standard deviation of relative parallax errors are 0.16 and 0.08 , respectively. The median value and standard deviation of proper motion errors are calculated 5.51 and 3.25 mas, respectively. Parallaxes and proper motion components are listed in Table 2 together with observational uncertainties. 

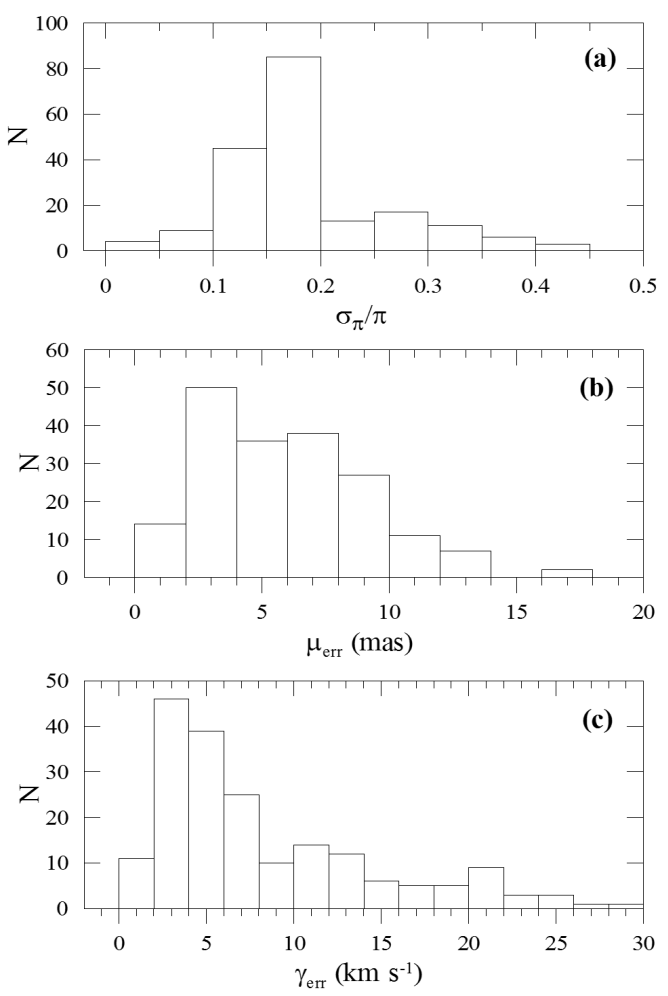

Fig. 1. Distribution of parallax errors (a), proper motion errors (b) and $\gamma$ velocity errors (c) of the present CV sample.

\subsection{Radial velocities}

Next necessary observational parameter to compute a space velocity of a star is its radial velocity with respect to Sun which comes from measurements of Doppler shifts of spectral lines. However, since CVs are binary stars their systemic velocities $(\gamma)$ are used. vPAS96 have collected systemic velocities of CVs from literature covering times up to the year 1994. For this study, systemic velocities of CVs published in the literature up to the middle of 2007 were collected in a similar style. It has been a convention to use $V_{r}(\phi)=$ $\gamma+K_{1,2} \sin \phi$ to predict systemic velocity of a $\mathrm{CV}$ from the measured radial velocities since orbits of CVs are circular, where $\phi$ is the orbital phase, $K_{1,2}$ are semi-amplitudes of radial velocity variation and 1 and 2 represent primary and secondary, respectively. $\gamma$ is the center of mass radial velocity of a $\mathrm{CV}$, which is usually found by non-linear least-square fit of the function to observed radial velocities.

The criteria defined by vPAS96 are adopted when collecting $\gamma$ velocities. Thus, if there are more than one determination of $\gamma$ for a $\mathrm{CV}$, the mean of existing $\gamma$ values is taken. A new mean value is computed similarly if there is new $\gamma$ velocity measurement which is not in vPAS96. Sometimes, more than one $\gamma$ value obtained with different methods are encountered, then the value rec- 
ommended by the author was adopted. The velocity measurements obtained during superoutbursts of SU UMa type dwarf novae have not been used since very large variations in $\gamma$ velocities have been observed during superoutbursts.

In the sample, $\gamma$ velocities of 59 systems (32 dwarf novae, 25 nova like stars, 1 nova and $1 \mathrm{CV}$ with unknown type) are from the absorption lines $\left(\gamma_{a b s}\right)$. Consequently, they are preferably used. However, it is well known that radial velocities derived from emission lines are likely affected by the motion in the accretion disc or the matter stream falling on the disc from the secondary. Thus, the measurements of $\gamma$ velocity obtained from emission lines $\left(\gamma_{e m}\right)$ may be unreliable (North et al., 2002). Radial velocities from the absorption lines of secondaries represent the system best. It should be noted that these very weak absorption lines are usually not observable in all CVs. They are most often visible in spectra of systems with orbital periods above the period gap (North et al., 2002).

For this study, we too look for possible systematic errors in the $\gamma_{e m}$ values and to provide information on the systematic and statistical accuracy of the $\gamma_{e m}$ values. In our data sample there are 47 systems ( 28 dwarf novae, 18 nova like stars and 1 nova) with both the $\gamma_{e m}$ and $\gamma_{a b s}$ values, from which we find an average difference $\left.<\gamma_{e m}-\gamma_{a b s}\right\rangle=+0.57 \pm 14.3 \mathrm{~km} \mathrm{~s}^{-1}$, where the error is the standard deviation of the distribution of individual differences. This is a result which is in agreement with vPAS96 who calculated an average difference $<\gamma_{e m}-\gamma_{a b s}>=+2.5 \pm 13.8 \mathrm{~km} \mathrm{~s}^{-1}$ using only 10 systems and concluded that there is no substantial systematic difference between systemic velocities derived from emission and absorption lines. Thus we too could conclude that the observed $\gamma$ velocities may allow a meaningful statistical analysis. Error histogram of $\gamma$ velocities is shown in Fig. 1c. The median value and standard deviation of $\gamma$ velocity errors are 5.6 and $6.3 \mathrm{~km} \mathrm{~s}^{-1}$, respectively. $\gamma$ velocities and associated uncertainties are listed in Table 2 together with distances and proper motion components.

\subsection{Galactic space velocities}

Space velocities with respect to Sun were computed using the algorithms and transformation matrices of Johnson \& Soderblom (1987). The input data (celestial coordinates $(\alpha, \delta)$, proper motion components $\left(\mu_{\alpha} \cos (\delta), \mu_{\delta}\right)$, systemic velocity $\gamma$ and the parallax $\pi$ ) are in the form adopted for the epoch of J2000 as described in the International Celestial Reference System (ICRS) of the Hipparcos and the Tycho Catalogues. Although sampled CVs are generally not distant objects, corrections for differential galactic rotation as described in Mihalas \& Binney (1981) were applied to the space velocities. 


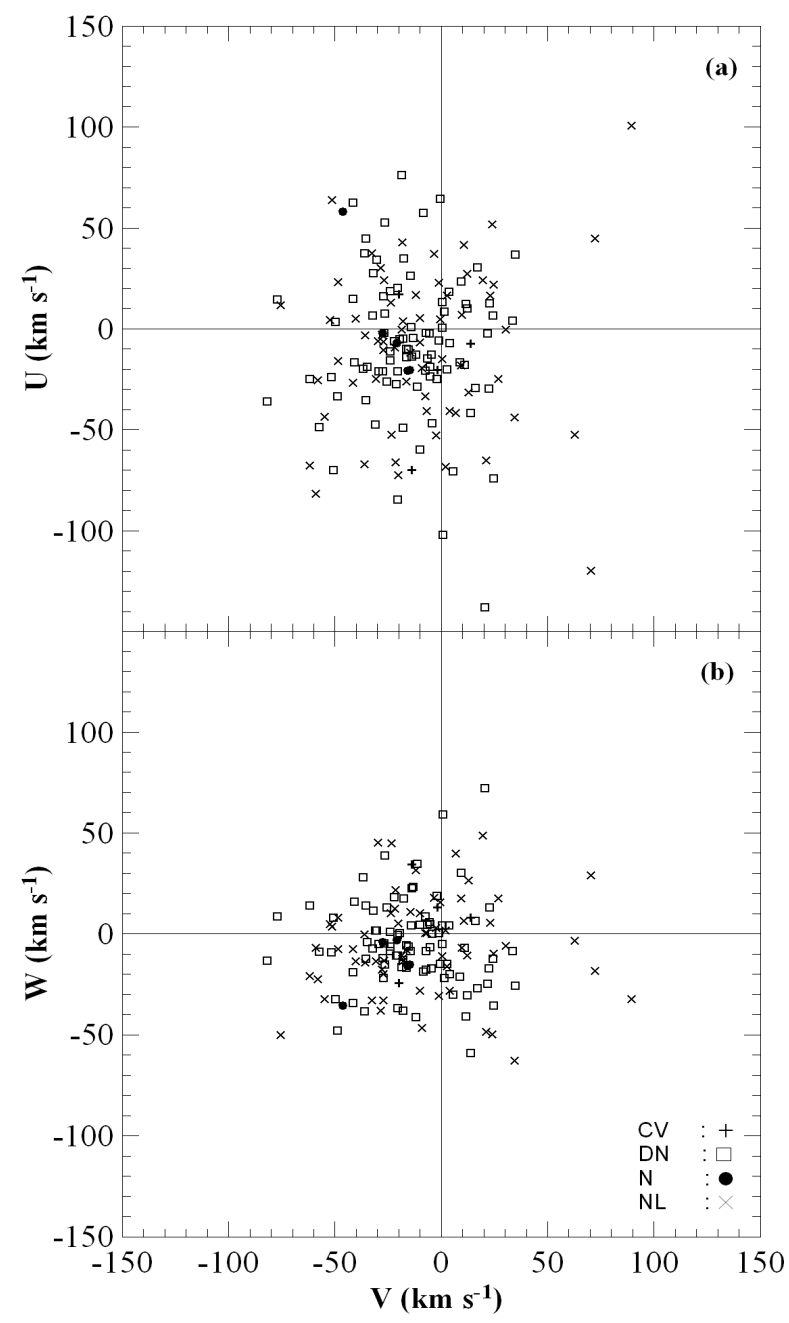

Fig. 2. Distribution of the space velocity components of CVs on the $U-V$ (a) and $W-V(\mathrm{~b})$ planes. CV denotes $\mathrm{CV}$ s with unknown types, DN dwarf novae, $\mathrm{N}$ novae and NL nova-like stars.

The transformation matrices of Johnson \& Soderblom (1987) use the notation of the right handed system. Therefore, the $U, V, W$ are the components of a velocity vector of a star with respect to the Sun, where the $U$ is directed toward the Galactic Center $\left(l=0^{\circ}, b=0^{\circ}\right)$; the $V$ is in the direction of the galactic rotation $\left(l=90^{\circ}, b=0^{\circ}\right)$; and the $W$ is towards the North Galactic Pole $\left(b=90^{\circ}\right)$.

The computed and corrected (galactic differential rotation) space velocity components are displayed in Fig. 2. Reliability of space velocities to indicate kinematical ages, the dispersion must be larger than the propagated errors. Therefore, the uncertainties of the space velocity components were also computed by propagating the uncertainties of the input data (proper motions, parallax and radial velocity) with an algorithm also by Johnson \& Soderblom (1987). The uncertainties of the space velocities $\left(S_{e r r}^{2}=U_{e r r}^{2}+V_{e r r}^{2}+W_{e r r}^{2}\right)$ were calculated, as well. Fig. 3 displays the histograms of the propagated un- 


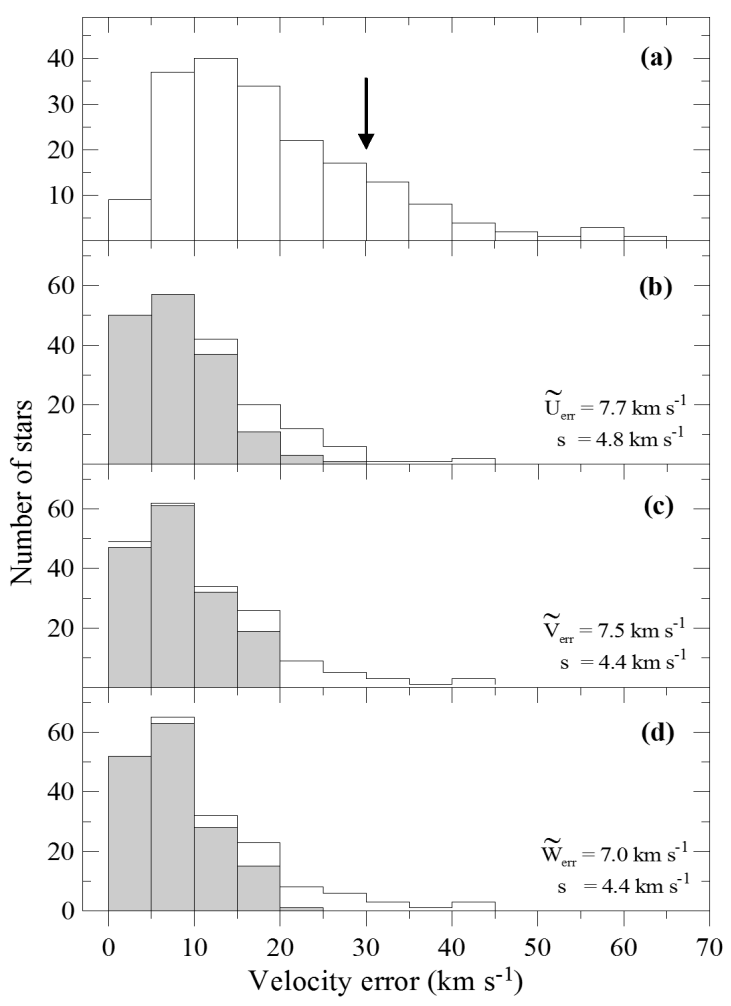

Fig. 3. Error histograms for space velocity vectors (a) and space velocity components (b-d) of CVs. The arrow indicates preferred $S_{\text {err }}$ limit.

certainties of space velocities $\left(S_{\text {err }}\right)$, and their components $\left(U_{\text {err }}, V_{\text {err }}, W_{\text {err }}\right)$. Apparently, very large uncertainties, which are bigger than $S_{e r r}>30 \mathrm{~km} \mathrm{~s}^{-1}$, exist not only for space velocity vectors but also among the components. A trial test showed us that removing some CVs with the largest space velocity errors reduces the dispersions of the space velocity as well as dispersion of its components $(U, V, W)$. Thus, we have decided to refine the sample by removing all CVs with $S_{\text {err }}>30 \mathrm{~km} \mathrm{~s}^{-1}$, which is the limit found by trial and error that shifting the $S_{\text {err }}$ limit further to a smaller value does not affect the space velocity dispersions. Thus, we have left 159 systems in the refined sample.

The $S_{\text {err }}$ limit and refined sample are indicated in Fig. 3 where the unshaded areas are the error distribution of the whole sample, and the shaded areas are the error distribution of the refined sample. The median values of the errors for the refined sample are $\widetilde{U}_{e r r}=7.7, \widetilde{V}_{e r r}=7.5$ and $\widetilde{W}_{e r r}=7.0 \mathrm{~km}$ $\mathrm{s}^{-1}$ while error distributions have standard deviations $\pm 4.8, \pm 4.4$ and \pm 4.4 $\mathrm{km} \mathrm{s}^{-1}$, respectively for the $U, V$ and $W$ components of the space velocity vectors. Such mean errors being smaller than dispersions on $U-V$ and $W-V$ diagrams (Fig. 2) indicate that the computed space velocity components have sufficient accuracy.

Further test of reliability is demonstrated in Fig. 4a-b that some systems as 


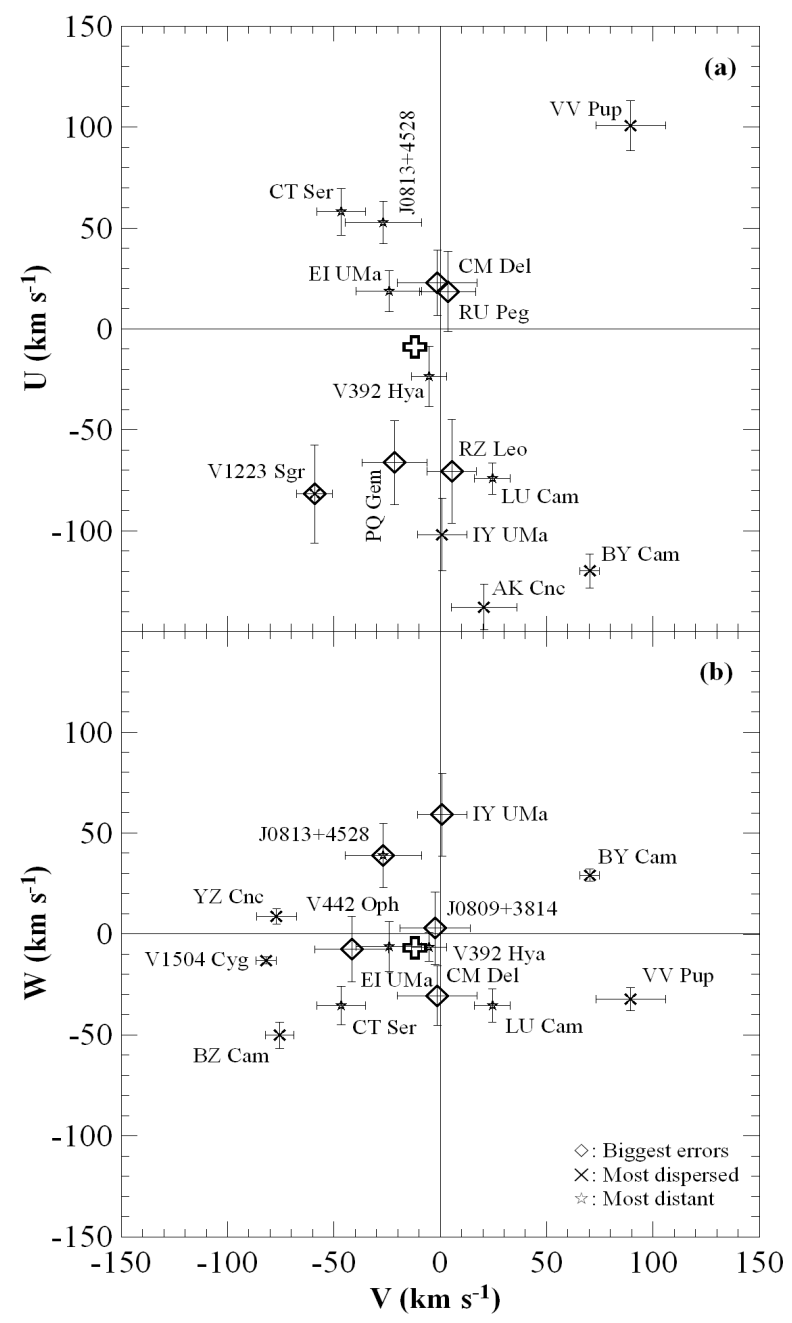

Fig. 4. The most distant five systems (stars), five systems with the biggest errors of space velocity vectors (diamonds), and five systems which are the most dispersed (times) on the $U-V$ and $W-V$ planes. The position of LSR is marked by a big-outlined plus symbol.

1) the most distant five systems, 2) five systems with the biggest errors of space velocity vectors, and 3) five systems which are the most dispersed on the $U-V$ and $W-V$ planes. The position for the local standard of rest (LSR) is known by subtracting the Sun's velocity $(U, V, W)_{\odot}=(9,12,7) \mathrm{km}$ $\mathrm{s}^{-1}$ (Mihalas \& Binney, 1981) from the frame of $(U, V, W)$ space velocities with respect to the Sun, and it is shown by a big outlined plus sign in Fig. $4 \mathrm{a}-\mathrm{b}$. One can see that the errors of the systems in the figure are not bigger than $\pm 26 \mathrm{~km} \mathrm{~s}^{-1}$ in both planes. Systems with biggest errors are not most dispersive ones. They are near the middle of the $U-V$ and $W-V$ planes. Fig. 4 indicates that dispersion in the velocity planes are unlikely to be affected by the velocity errors. Thus, the space velocities and the space velocity dispersions for the refined sample are reliable. 


\subsection{Space distributions}

Before interpreting space velocity dispersions, it is useful to investigate the space distribution of the sample in the solar neighbourhood. In order to inspect the spatial distribution of the refined sample, the Sun centered rectangular galactic coordinates $(X$ towards Galactic Centre, $Y$ galactic rotation, $Z$ North Galactic Pole) were computed. The projected positions on the galactic plane ( $X, Y$ plane) and on the plane perpendicular to it $(X, Z$ plane) are displayed in Fig. 5. Median distance of the whole sample is almost $300 \mathrm{pc}$ which is well within the galactic disc in the solar neighbourhood. Fig. 5 shows that different sub-groups of CVs in the sample share almost the same space in the solar neighbourhood. It should be noted that Ak et al. (2008) studied a CV sample of 459 systems including the present refined sample and found a median distance of $377 \mathrm{pc}$. A comparison shows that the refined sample of this study occupies nearly the same space of the sample used by Ak et al. (2008) despite the number of stars is three times less.

\subsection{Population analysis}

Population analysis was also needed in this study when investigating kinematical ages of some sub-groups. There are essentially two ways to find population types (thin and thick discs or halo) of solar neighbourhood field stars; the pure kinematical approach, or by looking at a combination of kinematics, metallicities, and stellar ages (Bensby et al., 2003). Here, we adopt the pure kinematical approach. Bensby et al. (2003) suggested a method to minimize the contamination of thick-disc stars with thin-disc stars. Their method is based on the assumption that the galactic space velocities of the stellar populations with respect to the LSR in the thin disc, the thick disc and the halo have Gaussian distributions,

$$
f(U, V, W)=k \cdot \exp \left(-\frac{U_{L S R}^{2}}{2 \sigma_{U_{L S R}}^{2}}-\frac{\left(V_{L S R}-V_{a s y m}\right)^{2}}{2 \sigma_{V_{L S R}}^{2}}-\frac{W_{L S R}^{2}}{2 \sigma_{W_{L S R}}^{2}}\right),
$$

where

$$
k=\frac{1}{(2 \pi)^{3 / 2} \sigma_{U_{L S R}} \sigma_{V_{L S R}} \sigma_{W_{L S R}}}
$$

normalizes the expression. $\sigma_{U_{L S R}}, \sigma_{V_{L S R}}$ and $\sigma_{W_{L S R}}$ are the characteristic velocity dispersions: 35,20 and $16 \mathrm{~km} \mathrm{~s}^{-1}$ for thin disc $(D) ; 67,38$ and $35 \mathrm{~km}$ $\mathrm{s}^{-1}$ for thick disc $(T D) ; 160,90$ and $90 \mathrm{~km} \mathrm{~s}^{-1}$ for halo $(H)$, respectively (Bensby et al., 2003). $V_{\text {asym }}$ is the asymmetric drift: $-15,-46$ and $-220 \mathrm{~km} \mathrm{~s}^{-1}$ 


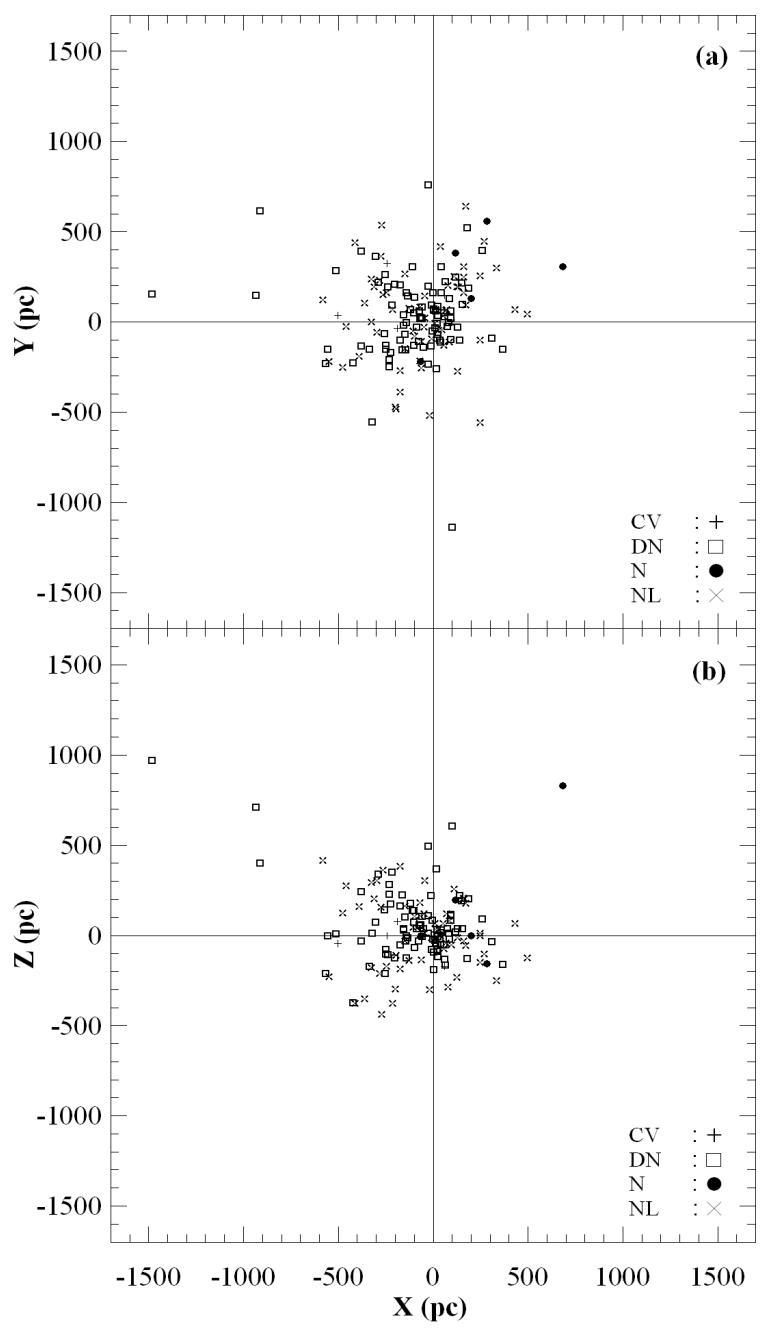

Fig. 5. The spatial distribution of CVs with respect to the Sun. $X, Y$ and $Z$ are heliocentric galactic coordinates directed towards the Galactic Centre, galactic rotation and the North Galactic Pole, respectively. Denotes are as in Fig. 3.

for thin disc, thick disc and halo, respectively. $U_{L S R}, V_{L S R}$ and $W_{L S R}$ are galactic space velocities with respect to the LSR. Space velocity components with respect to LSR are obtained by adding space velocities of CVs to the space velocity of Sun $\left((U, V, W)_{\odot}=(9,12,7) \mathrm{km} \mathrm{s}^{-1}\right.$, Mihalas \& Binney (1981) $)$ with respect to LSR.

In order to find the probability of a star belonging to a population, the probabilities from Eq. (1) has to be multiplied by the observed fractions $(X)$ of each population in the solar neighbourhood. Observed fractions of thin-disc, thick-disc and halo stars in the solar neighbourhood are adopted $X_{D}=0.94$, $X_{T D}=0.06$ and $X_{H}=0.0015$, respectively (Robin et al., 1996; Buser et al., 1999). Then, two relative probabilities for the thick disc to thin disc $(T D / D)$ and thick disc to halo $(T D / H)$ membership for each star are estimated as 
following

$$
T D / D=\frac{X_{T D}}{X_{D}} \cdot \frac{f_{T D}}{f_{D}}, \quad T D / H=\frac{X_{T D}}{X_{H}} \cdot \frac{f_{T D}}{f_{H}} .
$$

Stars are selected as from four different $T D / D$ intervals: $T D / D<0.1$ (i.e. "high probability thin-disc stars"); $0.1<T D / D<1$ (i.e. "low probability thin-disc stars"); $1<T D / D<10$ (i.e. "low probability thick-disc stars") and $T D / D>10$ (i.e. "high probability thick-disc stars") (Bensby et al., 2005).

$V_{L S R}-\log (T D / D)$ and Toomre diagrams of CVs in the refined sample are shown in Fig. 6. Horizontal lines divide the $V_{L S R}-\log (T D / D)$ diagram into four regions from bottom to the top; high probability thin-disc stars, low probability thin disc-stars, low probability thick-disc stars and high probability thick-disc stars. In the Toomre diagram of CVs, dashed circles delineate constant peculiar galactic space velocities $\left(U_{\underline{I} S R}^{2}+W_{I S R}^{2}\right)^{1 / 2}$ in steps of 50 $\mathrm{km} \mathrm{s}^{-1}$. According to the criteria given by Bensby et al. (2005), 131 systems $(\sim 83 \%)$ in the present refined CV sample are high probability thin-disc stars, 18 systems $(\sim 11 \%)$ low probability thin-disc stars, 5 systems $(\sim 3 \%)$ low probability thick-disc stars and 5 systems $(\sim 3 \%)$ high probability thick-disc stars. Thus, it can be concluded that the CVs in this study mostly belong to the thin disc population.

\subsection{Velocity dispersions and kinematical ages}

Kinematical ages of CV samples were calculated from their velocity dispersions by the well known relation of Cox (2000)

$$
\sigma_{\nu}^{3}(\tau)=\sigma_{\nu, \tau=0}^{3}+\frac{3}{2} \alpha_{V} \delta_{2} T_{\delta}\left[\exp \left(\frac{\tau}{T_{\delta}}\right)-1\right],
$$

where, $\sigma_{\nu, \tau=0}$ is the velocity dispersion at zero age, which is usually taken as 10 $\mathrm{km} \mathrm{s}^{-1}$ (Cox, 2000), $\alpha_{V}$ is a parameter describing the rotation curve $(\approx 2.95)$, $T_{\delta}$ is a time scale $\left(5 \times 10^{9} \mathrm{yr}\right), \delta_{2}$ is a diffusion coefficient $\left(3.7 \times 10^{-6}\left(\mathrm{~km} \mathrm{~s}^{-1}\right)^{3}\right.$ yr). $\sigma_{\nu}(\tau)$ is the total velocity dispersion of the group of CVs (sample). $\tau$ is the kinematical age of the group, which is to be computed. The total dispersion of space velocity vectors $\left(\sigma_{\nu}\right)$ is connected to the dispersion of the velocity components as

$$
\sigma_{\nu}^{2}=\sigma_{U_{L S R}}^{2}+\sigma_{V_{L S R}}^{2}+\sigma_{W_{L S R}}^{2}
$$

After computing $\sigma_{\nu}^{2}$ from the dispersions of velocity components, it is used in Eq. (4) for computing $\tau$. 


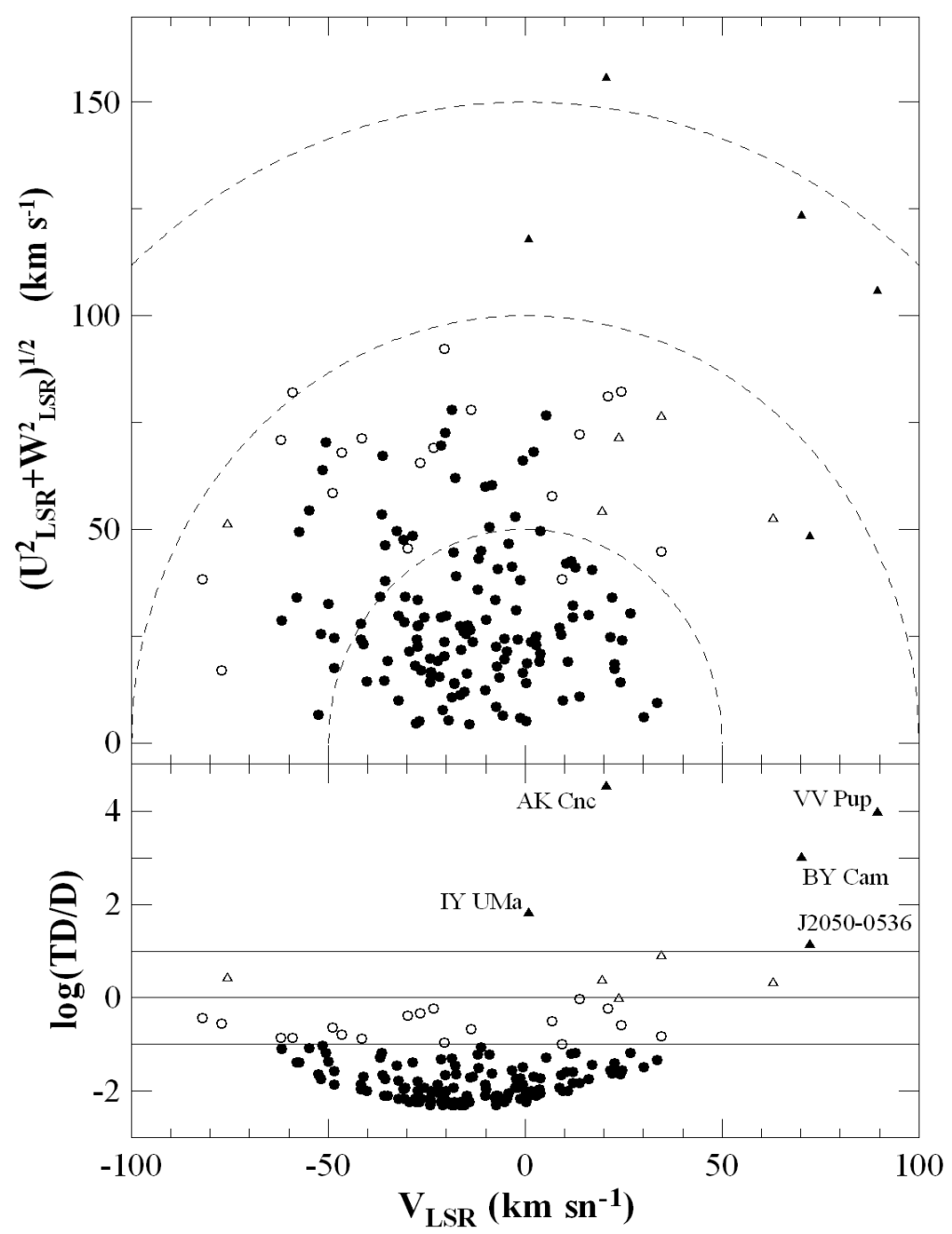

Fig. 6. Lower panel: $V_{L S R}-\log (T D / D)$ diagram of CVs. Filled and empty circles denote high and low probability thin-disc stars, while empty and filled triangles denote low and high probability thick-disc stars, respectively. Upper panel: Toomre diagram of the same CV sample.

For an isotropic distribution the $\gamma$ velocity dispersion is defined (Wielen et al., 1992; van Paradijs et al., 1996)

$$
\sigma_{\gamma}^{2}=\frac{1}{3} \sigma_{\nu}^{2}
$$

The $\gamma$ velocity dispersions are computed for a direct comparison to its theoretical predictions according to standard evolution model by Kolb \& Stehle (1996) and Kolb (2001), and listed in Table 3 for several sub-groups of CVs. 
Table 3

Mean space velocities, space velocity dispersions, kinematical ages and $\gamma$ velocity dispersions $\left(\sigma_{\gamma}\right)$ of CVs. TD/D<0.1 denotes high probability thin disc, $0.1<T D / D \leq 1$ low probability thin disc and $T D / D>1$ low and high probability thick disc. Nova-like stars in the table do not include magnetic systems (polars and intermediate polars). $\mathrm{N}$ is the number of systems.

\begin{tabular}{|c|c|c|c|c|c|c|c|c|c|c|}
\hline Parameter & $N$ & $\begin{array}{c}<U_{L S R}> \\
\left(\mathrm{km} \mathrm{s}^{-1}\right)\end{array}$ & $\begin{array}{c}<V_{L S R}> \\
\left(\mathrm{km} \mathrm{s}^{-1}\right)\end{array}$ & $\begin{array}{c}<W_{L S R}> \\
\left(\mathrm{km} \mathrm{s}^{-1}\right)\end{array}$ & $\begin{array}{c}\sigma_{U_{L S R}} \\
\left(\mathrm{~km} \mathrm{~s}^{-1}\right)\end{array}$ & $\begin{array}{c}\sigma_{V_{L S R}} \\
\left(\mathrm{~km} \mathrm{~s}^{-1}\right)\end{array}$ & $\begin{array}{c}\sigma_{W_{L S R}} \\
\left(\mathrm{~km} \mathrm{~s}^{-1}\right)\end{array}$ & $\begin{array}{c}\sigma_{\nu} \\
\left(\mathrm{km} \mathrm{s}^{-1}\right)\end{array}$ & $\begin{array}{l}\text { Age } \\
(\mathrm{Gyr})\end{array}$ & $\begin{array}{c}\sigma_{\gamma} \\
\left(\mathrm{km} \mathrm{s}^{-1}\right)\end{array}$ \\
\hline All sample & 159 & $+0.07 \pm 2.93$ & $-0.48 \pm 2.19$ & $+1.53 \pm 1.80$ & $36.86 \pm 4.78$ & $27.48 \pm 4.45$ & $22.69 \pm 3.65$ & $51.27 \pm 7.48$ & $4.84 \pm 1.37$ & $30 \pm 4$ \\
\hline Nova-like stars & 38 & $+1.67 \pm 5.20$ & $-1.63 \pm 4.72$ & $+0.97 \pm 4.07$ & $31.67 \pm 3.86$ & $28.74 \pm 4.65$ & $24.79 \pm 4.36$ & $49.43 \pm 7.45$ & $4.51 \pm 1.36$ & $29 \pm 4$ \\
\hline Novae & 5 & $+10.49 \pm 14.59$ & $-13.16 \pm 5.81$ & $-7.64 \pm 5.87$ & $31.00 \pm 3.23$ & $17.56 \pm 3.38$ & $14.01 \pm 2.13$ & $38.28 \pm 5.14$ & $2.57 \pm 0.86$ & $22 \pm 3$ \\
\hline Non-magnetic systems & 134 & $+0.80 \pm 2.96$ & $-2.29 \pm 2.13$ & $+1.46 \pm 1.96$ & $34.11 \pm 4.57$ & $24.68 \pm 4.53$ & $22.61 \pm 4.41$ & $47.79 \pm 7.80$ & $4.21 \pm 1.42$ & $28 \pm 5$ \\
\hline$T D / D<0.1$ & 131 & $+2.97 \pm 2.49$ & $-2.37 \pm 1.81$ & $+1.34 \pm 1.46$ & $28.60 \pm 4.68$ & $20.76 \pm 4.48$ & $16.65 \pm 4.39$ & $39.07 \pm 7.83$ & $2.70 \pm 1.35$ & $23 \pm 5$ \\
\hline $0.1<T D / D \leq 1$ & 18 & $-13.59 \pm 12.09$ & $-11.41 \pm 8.43$ & $+0.24 \pm 8.66$ & $51.67 \pm 5.64$ & $36.57 \pm 3.91$ & $35.69 \pm 4.73$ & $72.67 \pm 8.33$ & $8.68 \pm 1.38$ & $42 \pm 5$ \\
\hline$T D / D>1$ & 10 & $-13.34 \pm 25.60$ & $+43.88 \pm 15.00$ & $+6.24 \pm 15.73$ & $77.95 \pm 4.30$ & $62.85 \pm 4.87$ & $47.59 \pm 4.71$ & $110.87 \pm 8.02$ & $14.35 \pm 0.99$ & $64 \pm 5$ \\
\hline$P(h)<2.62$ & 62 & $+0.46 \pm 4.82$ & $+1.38 \pm 3.89$ & $+8.29 \pm 3.16$ & $37.64 \pm 5.10$ & $30.45 \pm 4.41$ & $26.02 \pm 4.53$ & $54.96 \pm 8.12$ & $5.52 \pm 1.48$ & $32 \pm 5$ \\
\hline $3.35<P(h) \leq 4.50$ & 39 & $-2.01 \pm 6.55$ & $-1.12 \pm 4.66$ & $-3.67 \pm 3.70$ & $40.41 \pm 4.47$ & $28.72 \pm 4.15$ & $23.07 \pm 3.89$ & $54.68 \pm 7.23$ & $5.47 \pm 1.32$ & $32 \pm 4$ \\
\hline $4.50<P(h) \leq 11.00$ & 39 & $+1.95 \pm 5.90$ & $-4.39 \pm 3.56$ & $-1.83 \pm 2.99$ & $36.44 \pm 4.69$ & $22.40 \pm 4.51$ & $18.54 \pm 4.23$ & $46.62 \pm 7.76$ & $4.00 \pm 1.41$ & $27 \pm 4$ \\
\hline
\end{tabular}


Table 4

Mean space velocities, space velocity dispersions, kinematical ages and $\gamma$ velocity dispersions $\left(\sigma_{\gamma}\right)$ of non-magnetic systems according to the orbital period ranges. $\mathrm{N}$ is the number of CVs.

\begin{tabular}{|c|c|c|c|c|c|c|c|c|c|c|}
\hline Parameter & $N$ & $\begin{array}{c}<U_{L S R}> \\
\left(\mathrm{km} \mathrm{s}^{-1}\right)\end{array}$ & $\begin{array}{c}<V_{L S R}> \\
\left(\mathrm{km} \mathrm{s}^{-1}\right)\end{array}$ & $\begin{array}{c}<W_{L S R}> \\
\left(\mathrm{km} \mathrm{s}^{-1}\right)\end{array}$ & $\begin{array}{c}\sigma_{U_{L S R}} \\
\left(\mathrm{~km} \mathrm{~s}^{-1}\right)\end{array}$ & $\begin{array}{c}\sigma_{V_{L S R}} \\
\left(\mathrm{~km} \mathrm{~s}^{-1}\right)\end{array}$ & $\begin{array}{c}\sigma_{W_{L S R}} \\
\left(\mathrm{~km} \mathrm{~s}^{-1}\right)\end{array}$ & $\begin{array}{c}\sigma_{\nu} \\
\left(\mathrm{km} \mathrm{s}^{-1}\right)\end{array}$ & $\begin{array}{l}\text { Age } \\
(\mathrm{Gyr})\end{array}$ & $\begin{array}{c}\sigma_{\gamma} \\
\left(\mathrm{km} \mathrm{s}^{-1}\right)\end{array}$ \\
\hline$P(h)<2.62$ & 53 & $-2.47 \pm 4.98$ & $-2.25 \pm 3.73$ & $+8.10 \pm 3.50$ & $35.97 \pm 5.22$ & $26.99 \pm 4.33$ & $26.49 \pm 4.45$ & $52.19 \pm 8.11$ & $5.01 \pm 1.48$ & $30 \pm 5$ \\
\hline$P(h) \geq 2.62$ & 81 & $+2.94 \pm 3.66$ & $-2.31 \pm 2.56$ & $-2.89 \pm 2.17$ & $32.84 \pm 4.06$ & $23.04 \pm 4.59$ & $19.65 \pm 4.22$ & $44.67 \pm 7.44$ & $3.65 \pm 1.34$ & $26 \pm 4$ \\
\hline $1.06<P(h) \leq 1.80$ & 34 & $-6.60 \pm 5.81$ & $-3.23 \pm 4.61$ & $+6.88 \pm 4.22$ & $34.04 \pm 4.36$ & $26.68 \pm 4.72$ & $25.21 \pm 4.79$ & $50.06 \pm 8.01$ & $4.62 \pm 1.47$ & $29 \pm 5$ \\
\hline $1.80<P(h) \leq 3.35$ & 33 & $+2.19 \pm 6.06$ & $+0.38 \pm 4.70$ & $+3.41 \pm 4.20$ & $34.35 \pm 5.23$ & $26.57 \pm 4.46$ & $24.01 \pm 4.43$ & $49.62 \pm 8.18$ & $4.54 \pm 1.50$ & $29 \pm 5$ \\
\hline $3.35<P(h) \leq 4.60$ & 33 & $+0.90 \pm 6.23$ & $-3.65 \pm 4.33$ & $-2.33 \pm 3.66$ & $35.23 \pm 3.78$ & $24.76 \pm 4.38$ & $20.84 \pm 4.01$ & $47.84 \pm 7.04$ & $4.22 \pm 1.29$ & $28 \pm 4$ \\
\hline $4.60<P(h) \leq 11.00$ & 33 & $+6.17 \pm 5.73$ & $-2.68 \pm 3.60$ & $-3.41 \pm 3.32$ & $33.01 \pm 4.42$ & $20.52 \pm 4.61$ & $19.09 \pm 4.14$ & $43.30 \pm 7.61$ & $3.41 \pm 1.36$ & $25 \pm 4$ \\
\hline
\end{tabular}




\section{Discussions}

Except four systems (V392 Hya, CT Ser, EI UMa and J0813+4528) all of the refined sample are contained within $500 \mathrm{pc}$ from the galactic plane. Thus, all of the CVs in the refined sample could be considered within the galactic disc (Fig. 5). Concentration towards the Sun and sparseness outwards indicate that space distribution of present data is highly affected by selection with respect to brightness.

Various sub-groups of the refined sample have been formed to investigate if there are kinematically homogeneous sub-systems. All sample and outstanding sub-groups have been summarized together with their mean space velocity components, velocity dispersions of the components, total dispersion and corresponding kinematical age of the group according to Eq. (4) in Tables 3 and 4. The velocity dispersions in Table $3\left(\sigma_{U_{L S R}}=36.86 \mathrm{~km} \mathrm{~s}^{-1}, \sigma_{V_{L S R}}=27.48\right.$ $\left.\mathrm{km} \mathrm{s}^{-1}, \sigma_{W_{L S R}}=22.69 \mathrm{~km} \mathrm{~s}^{-1}\right)$ indicate that the present refined sample has a mean kinematical age of $4.84 \pm 1.37$ Gyr. Considering the total dispersion $\left(\sigma_{\nu}=51.27 \mathrm{~km} \mathrm{~s}^{-1}\right)$ for the refined sample as a whole, its empirical $\gamma$ velocity dispersion is $\sigma_{\gamma}=30 \pm 4 \mathrm{~km} \mathrm{~s}^{-1}$ according to Eq. (6).

\subsection{Kinematics of $C V$ types}

Kinematical properties of standard sub-groups; dwarf novae (DN), nova-like systems (NL) and novae were investigated first. Those sub-groups were shown with different symbols on the $U-V$ and $W-V$ diagrams in Fig. 2. Their kinematics were summarized in Table 3. There is no clear distinction between the distributions of first two sub-groups on the velocity space. Mean space velocities and velocity dispersions of dwarf novae and nova-like stars appear similar with slightly older kinematical age of nova-like stars. Although their number is statistically insignificant, novae appear to be the youngest $(2.57 \pm$ 0.86 Gyr). Note that Ak et al. (2008) found novae in their sample as located almost on the galactic plane, suggesting that they are young systems.

Comparisons of $U-V$ diagrams of dwarf novae, nova-like stars and novae are shown in Fig. 7. It should be noted that magnetic systems (polars and intermediate polars) are removed from the sample since the evolution of magnetic systems can be different than the evolution of non-magnetic CVs (Wu \& Wickramasinghe, 1993; Webbink \& Wickramasinghe, 2002; Schwarz et al., 2007). Central concentration of considerable amount of dwarf novae towards LSR makes them appear slightly younger while a central concentration in the $U-V$ diagram of nova-like stars is not observed.

Magnetic and non-magnetic systems display different kinematical properties 


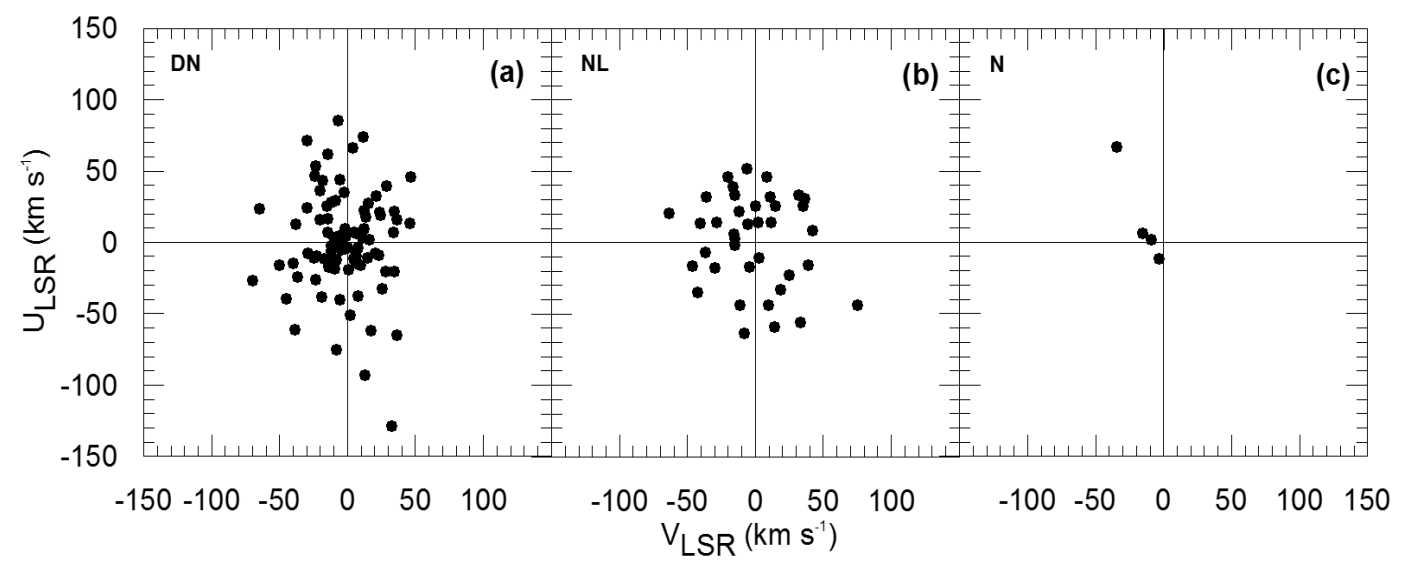

Fig. 7. $U-V$ diagrams of CVs for sub-types. DN denotes dwarf novae (a), NL nova-like stars (b) and N novae (c). Magnetic systems are not included in the sample.

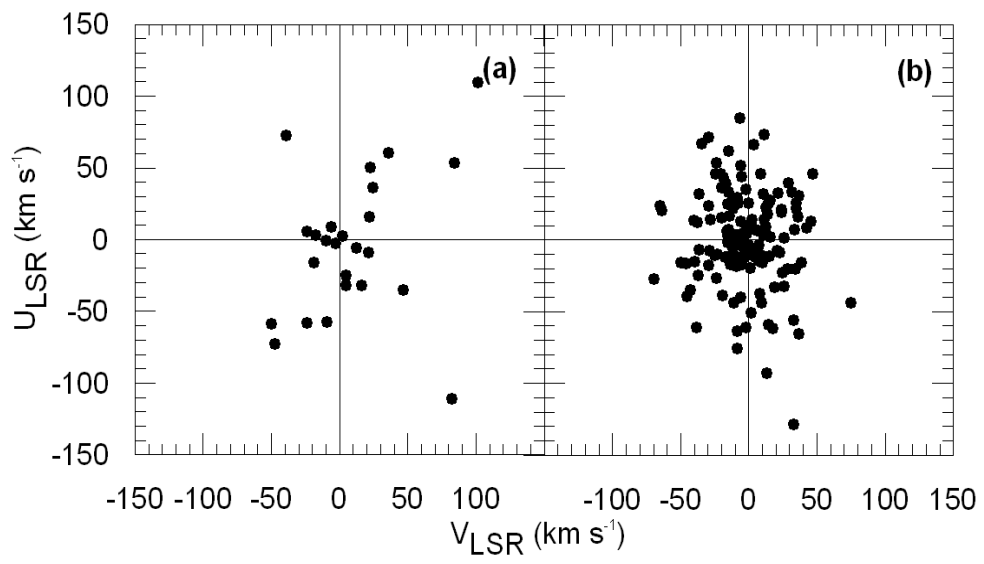

Fig. 8. $U-V$ diagrams of magnetic (a) and non-magnetic CVs (b).

(Table 3). Although the number of magnetic systems is small $(\mathrm{N}=25)$ which is near the limit of statistical significance, they appear almost twice older than non-magnetic systems. Concentration towards LSR which makes the dispersion smaller for non-magnetic systems is clear in Fig. 8b. However, $\gamma$ velocities derived for magnetic CVs may be significantly contaminated by the flow velocities of the magnetically channelled plasma. Thus, their $\gamma$ velocities may not be reliable. Consequently, kinematically older appearance may not be the truth.

\subsection{Groups according to population analysis}

Since population analysis is directly related to kinematical properties of stars, the distribution of the groups according to the population analysis on the $U-V$ diagram show very distinct properties (see Fig. 9 and Table 3). It is inconsistent to assign a kinematical age to these groups, because formation of 


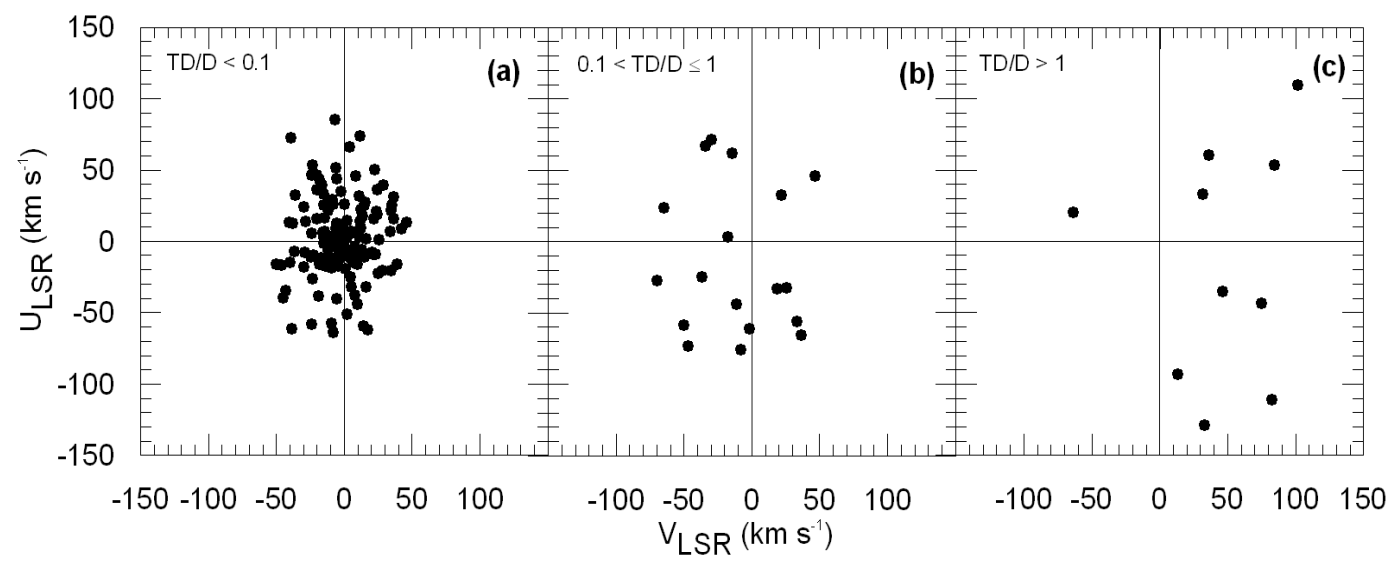

Fig. 9. $U-V$ diagrams of $\mathrm{CV}$ populations. $T D / D<0.1$ shows high probability thin-disc stars (a), while $0.1<T D / D \leq 1$ shows low probability thin-disc stars (b), and $T D / D>1$ represents both low and high probability thick-disc stars (c).

groups is already according to their kinematics.

Among the 131 high probability thin-disc $(T D / D<0.1) \mathrm{CVs}$, the most are dwarf novae, with 33 SU UMa type stars, 21 U Gem type stars, 12 Z Cam type stars and nine unknown type dwarf novae. All Z Cam type dwarf novae are found among the high probability thin-disc stars. Among high probability thin-disc CVs, four are novae and three are unknown type. Number of novalike stars in high probability thin-disc CVs is 49, with one AC Cnc type star, eight polars, nine intermediate polars, 16 UX UMa type stars, five VY Scl type stars and one unknown type nova-like star. Although magnetic systems appear to be old in Fig. 8, 17 of them are contained in high probability thin disc group in Fig. 9 which indicates youth.

Among the low probability thin-disc $(0.1<T D / D \leq 1) \mathrm{CVs}$, the 10 of them are dwarf novae (four SU UMa type stars, four U Gem type stars and two unknown type dwarf novae), six of them are nova-like stars (one polar, two intermediate polars, two UX UMa type stars and one unknown type nova-like star), one is nova and one is unknown type.

Nova-like stars comprise the majority of low and high probability thick-disc stars $(T D / D>1)$. Among 10, eight of them are nova-like stars, with four polars, one intermediate polar, one VY Scl star and two unknown type. Only two of 10 are dwarf novae.

In this study we find that almost all of CVs in the refined sample could be considered within the galactic disc (Fig. 5). Although there are relatively a few thick-disc and halo stars in solar neighbourhood, thick-disc and halo stars are dominant in $z$-distance intervals $1.5<z \leq 5$, and $z>5 \mathrm{kpc}$, respectively (Karaali et al., 2004; Bilir et al., 2006; Ak et al., 2007b; Bilir et al., 2008). Here, $z$ denotes vertical distance from the galactic plane. Thus, thick- 


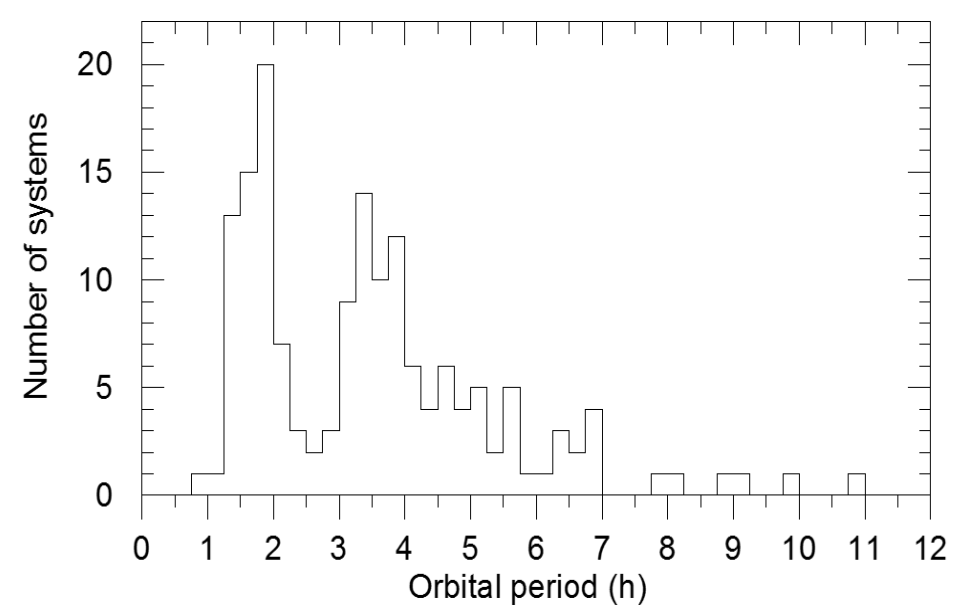

Fig. 10. Orbital period distribution of CVs in the refined sample.

disc and halo CVs must be the most distant systems. They can be detected by using the deep photometric systems, e.g. SDSS, IPHAS, etc.

\subsection{Groups according to orbital periods}

Since the orbital period of a CV is directly related to the mean mass density of the secondary star, which is supposed to be an age related quantity, investigating kinematical properties according to orbital period ranges could be the most meaningful. Moreover, orbital period evolution of CV samples has been predicted by various authors (see introduction).

In order to search kinematical differences among the sub-groups of limited orbital period ranges, first, distribution of orbital periods must be studied. The orbital period distribution of the refined sample is shown in Fig. 10 where the orbital period gap is distinctly visible between $2 \mathrm{~h}$ and $3 \mathrm{~h}$. Separating point of the period gap is found at $2.62 \mathrm{~h}$ by fitting Gaussians with two peaks to the orbital period distribution in Fig. 10. It should be emphasized that this approach is just one way to define the middle of the gap.

The $U-V$ diagrams of the sub-groups of $P(h)<2.62$ and $P(h) \geq 2.62$ are shown in Fig. 11 and their kinematics are given in Table 3. Table 3 indicate that systems below the period gap $(P(h)<2.62)$ are kinematically older than systems above the gap $(P(h) \geq 2.62)$ as predicted by standard model. However, a comparison between the two distributions indicates $U-V$ diagram and other kinematical quantities are almost the same for these sub-groups. Similarity is indicated by that both groups have central concentrations (low dispersion stars) as well as high velocity stars. If one considers kinematical ages are the same within the error limits, it can be concluded that the result would be in agreement with vPAS96. 


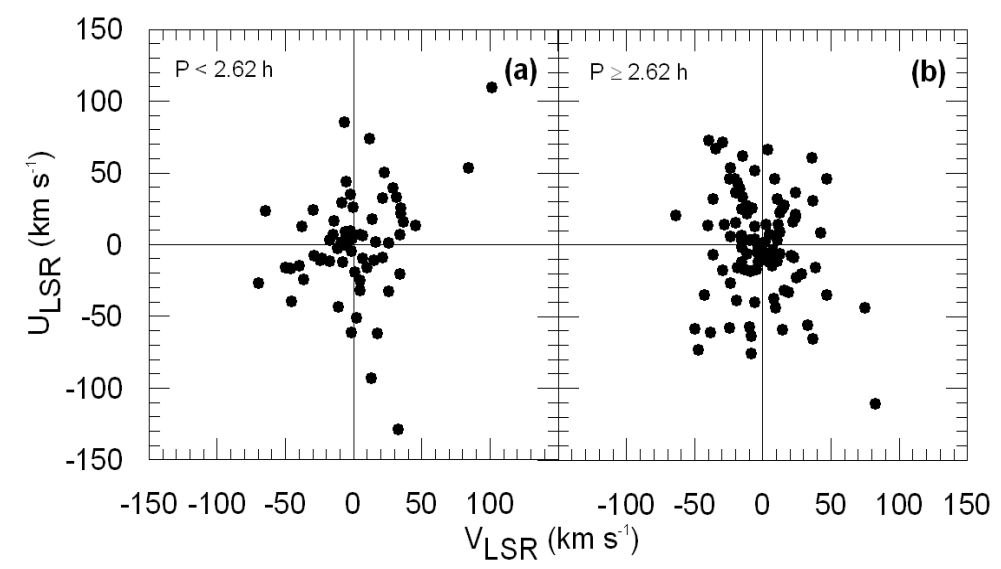

Fig. 11. $U-V$ diagrams of CVs below (a) and above (b) the orbital period gap.

Another way to investigate different period groups is to divide the sample according to the same number of CVs at different period ranges. Such a grouping is made and summarized in the last four lines of Table 3. Table 3 shows that there is an excess of the space velocity dispersion in $3.35<P(h) \leq 4.50$ period interval. This is mainly due to magnetic systems (polars and intermediate polars) whose $\gamma$ velocities may not be reliable, as pointed above. Thus, magnetic systems are removed from the refined sample, leaving 134 non-magnetic CVs in the sample.

After removing the magnetic systems, the sample analyzed in a similar style. When comparing the kinematics of non-magnetic CVs below and above the period gap (Table 4 and Fig. 12), it has been found that difference of kinematical ages slightly incereased as indicated by dispersions. On the other hand, kinematics and $U-V$ diagrams of the four sub-groups without magnetic systems are summarized in the last four lines of Table 4. It becomes clear after removing magnetic systems from the sample that towards the longer periods dispersions become smaller with younger ages. The $U-V$ diagrams of four sub-groups of non-magnetic systems are shown in Fig. 13. It should be noted that for the systems grouped in Fig. $13 \gamma$ velocity is increasingly difficult to measure from panel (d) to (a).

Table 4 shows that the mean kinematical age difference between the nonmagnetic systems above and below the orbital period gap is about 1.4 Gyr. Thus, kinematics of the present sample of CVs roughly confirm the prediction of Kolb \& Stehle (1996) who predicted about 2 Gyr age difference between the $\mathrm{CV}$ groups of below and above the period gap. 


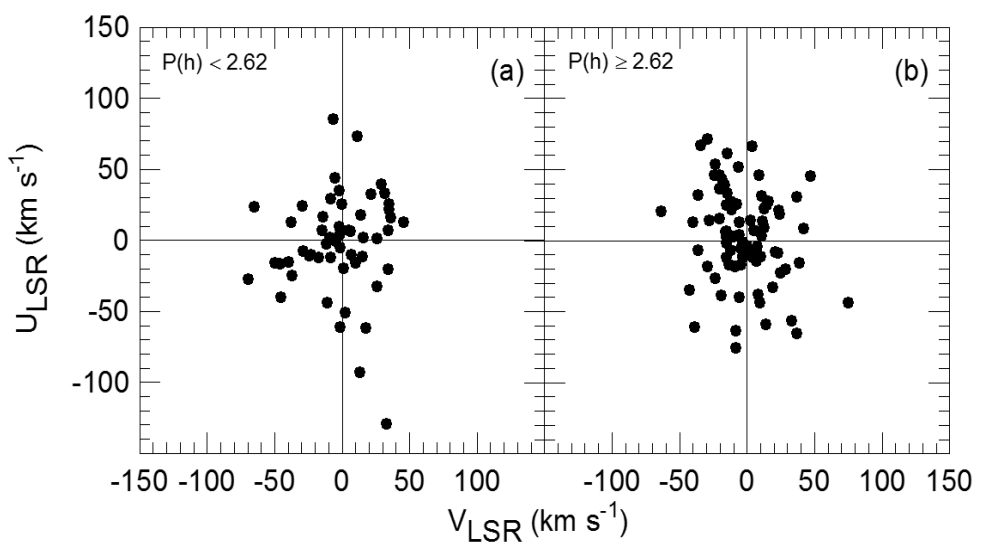

Fig. 12. $U-V$ diagrams of non-magnetic CVs below $(P(h)<2.62)$ and above $(P(h) \geq 2.62)$ the orbital period gap.

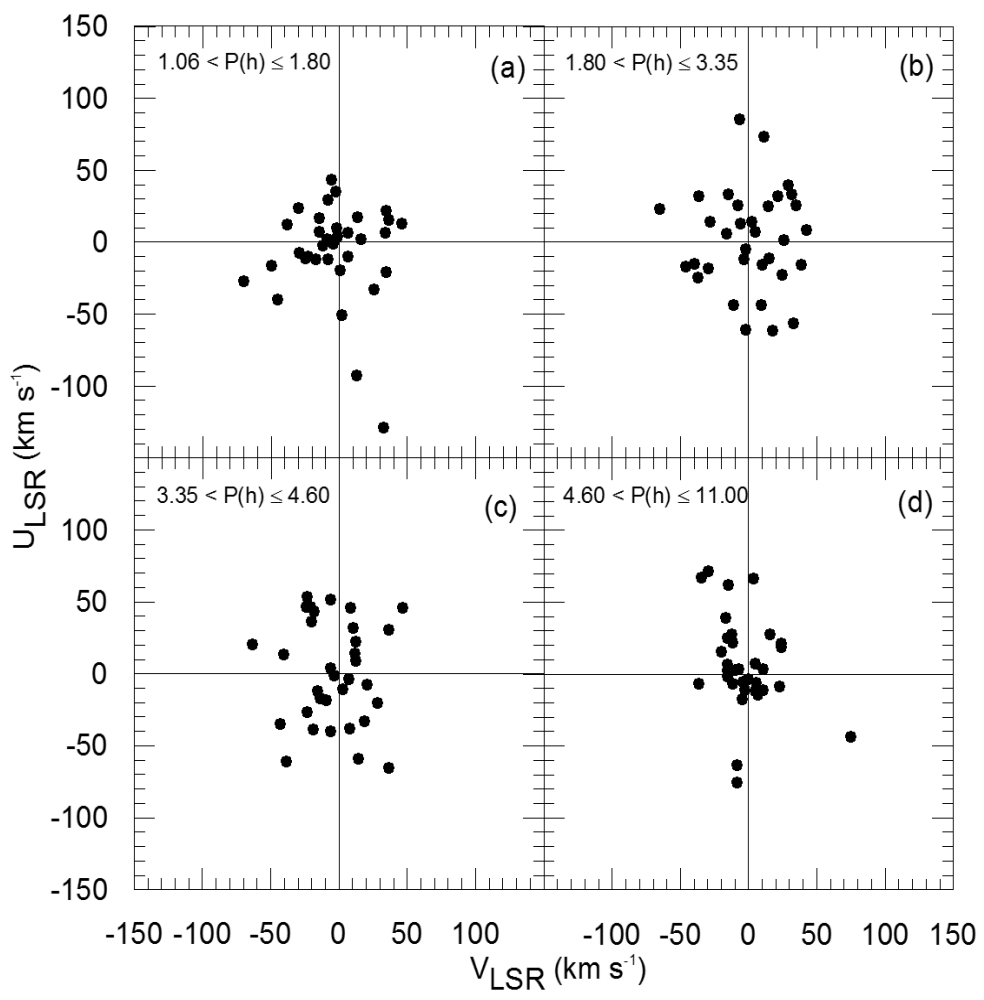

Fig. 13. $U-V$ diagrams of non-magnetic $\mathrm{CVs}$ in different orbital period ranges.

\section{$3.4 \gamma$ velocity dispersion of $C V s$}

The definition of $\gamma$ velocity dispersion in Eq. (6) is based on the assumption that the distribution of space velocity components is isotropic (Wielen et al., 1992; van Paradijs et al., 1996). However, the truth is that it is not isotropic as $\sigma_{U_{L S R}}$ is always higher than $\sigma_{V_{L S R}}$ and $\sigma_{W_{L S R}}$ (see Table 3 and 4 ). Nevertheless, $\gamma$ velocity dispersion of a $\mathrm{CV}$ sub-group calculated from total space velocity dispersion $\left(\sigma_{\nu}\right)$ as in Eq. (6) is useful for direct comparison of theoretical 
predictions.

The discussions of $\gamma$ velocity dispersions are based on non-magnetic systems. $\gamma$ velocity dispersion of all non-magnetic systems in the refined sample is estimated $\sigma_{\gamma}=28 \pm 5 \mathrm{~km} \mathrm{~s}^{-1}$. For a comparison with theoretical predictions, $\gamma$ velocity dispersions of non-magnetic CVs below $(P(h)<2.62)$ and above $(P(h) \geq 2.62)$ the period gap are calculated $\sigma_{\gamma}=30 \pm 5 \mathrm{~km} \mathrm{~s}^{-1}$ and $\sigma_{\gamma}=26 \pm 4$ $\mathrm{km} \mathrm{s}^{-1}$ (see Table 4), respectively. Theoretical predictions of $\gamma$ velocity dispersion are $\sigma_{\gamma} \simeq 30 \mathrm{~km} \mathrm{~s}^{-1}$ and $\sigma_{\gamma} \simeq 15 \mathrm{~km} \mathrm{~s}^{-1}$ (Kolb \& Stehle, 1996) for below and above the gap, respectively. Although the observational $\gamma$ velocity dispersion of the systems below the period gap is in agreement with its theoretical prediction given by Kolb \& Stehle (1996), the observational $\gamma$ velocity dispersion of the systems above the gap is not in agreement with its theoretical

prediction. However, Kolb (2001) suggested that the $\gamma$ velocity dispersions of $\mathrm{CVs}$ are $\simeq 32 \mathrm{~km} \mathrm{~s}^{-1}$ below the period gap versus $\simeq 27 \mathrm{~km} \mathrm{~s}^{-1}$ above the gap, respectively, if magnetic braking does not operate in the detached phase, during which the system evolves from the post-CE orbit into contact. It seems that our results confirm Kolb (2001).

It should be noted that cumulative effect of repeated nova explosions with possibly asymmetric envelope ejection would increase the $\gamma$ velocity dispersions of CVs during their evolution (Kolb \& Stehle, 1996). In this case, the observational $\gamma$ velocity dispersions in this study would represent upper limits while theoretical values would be underestimated. Thus, difference between observational and theoretical values increases. In addition, if nova explosions increased the $\gamma$ velocity dispersions of CVs during their evolution, kinematical ages found in our study would also represent upper limits. However, nova explosions can not affect the age and $\gamma$ velocity dispersion differences between sub-groups of CVs.

\section{Conclusions}

The $\gamma$ velocities of CVs are taken from a variety of sources from the literature. For all but 59 systems, published $\gamma$ velocities are by-products of measurements of the components' semi-amplitude. In addition, measurements based on emission lines remain problematic. As a consequence, systematic errors affect specifically the determination of $\gamma$ velocity and error values are high.

After analysing available kinematical data of CVs, it is concluded that there is not considerable kinematical difference between dwarf novae and non-magnetic nova-like stars. Magnetic and non-magnetic systems display different kinematical properties. However, kinematics based on $\gamma$ velocity measurements of magnetic systems remain problematic as their $\gamma$ velocities may be significantly 
contaminated by the flow velocities of the magnetically channelled plasma.

Kinematics of the present sample show that systems above the orbital pediod gap are younger than systems below the gap. This result is in agreement with the standard theory of the CV evolution. Kinematics of the present sample of CVs also roughly confirm the prediction of Kolb \& Stehle (1996) who predicted about 2 Gyr age difference between the CV groups of below and above the period gap. Smaller age difference found in this study shows a similarity of the angular momentum loss time scales in systems with low-mass and high-mass secondary stars (Kolb, 2001).

Assuming an isotropic distribution, observational $\gamma$ velocity dispersions of CVs are in agreement with the theoretical predictions of Kolb (2001) who suggested that the difference of $\gamma$ velocity dispersions of the systems below and above the period gap is about $5 \mathrm{~km} \mathrm{~s}^{-1}$ with systems above the gap having a $\gamma$ velocity dispersion of $\simeq 27 \mathrm{~km} \mathrm{~s}^{-1}$. This agreement between the observations and the theory implies that magnetic braking does not operate in the detached phase.

\section{Acknowledgments}

Part of this work was supported by the Research Fund of the University of Istanbul, Project Number: BYP-1379. This research has made use of the SIMBAD database, operated at CDS, Strasbourg, France. This publication makes use of data products from the Two Micron All Sky Survey, which is a joint project of the University of Massachusetts and the Infrared Processing and Analysis Center/California Institute of Technology, funded by the National Aeronautics and Space Administration and the National Science Foundation. This research has made use of the NASA/IPAC Extragalactic Database (NED) which is operated by the Jet Propulsion Laboratory, California Institute of Technology, under contract with the National Aeronautics and Space Administration. We thank the anonymous referee for a thorough report and useful comments that helped improving an early version of the paper.

\section{References}

Adelman-McCarthy, J.K., et al., 2006. ApJS 162, 38.

Ak, T., Bilir, S., Ak, S., Retter, A., 2007a. NewA 12, 446.

Ak, S., Bilir, S., Karaali, S., Buser, R., 2007b. AN 328, 169.

Ak, T., Bilir, S., Ak, S., Eker, Z., 2008. NewA 13, 133.

Andronov, N., Pinsonneault, M., Sills, A., 2003. ApJ 582, 358.

Arenas, J., Mennickent, R.E., 1998. A\&A 337, 472.

Arenas, J., Catalán, M.S., Augusteijn, T., Retter, A., 2000. MNRAS 311, 135. 
Augusteijn, T., Wisotzki, L., 1997. A\&A 324, L57.

Aungwerojwit, A., Gänsicke, B.T., Rodríguez-Gil, P., Hagen, H.-J., Harlaftis,

E.T., Papadimitriou, C., Lehto, H., Araujo-Betancor, S., Heber, U., Fried,

R.E., Engels, D., Katajainen, S., 2005. A\&A 443, 995.

Baptista, R., Catalan, M.S., Costa, L., 2000. MNRAS 316, 529.

Belle, K.E., Howell, S.B., Mukai, K., Szkody, P., Nishikida, K., Ciardi, D.R.,

Fried, R.E., Oliver, J.P., 2005. AJ 129, 1985.

Bensby, T., Feltzing, S., Lundström, I., 2003. A\&A 410, 527.

Bensby, T., Feltzing, S., Lundström, I., Ilyin, I., 2005. A\&A 433, 185.

Beuermann, K., Harrison, T.E., McArthur, B.E., Benedict, G.F., Gänsicke, B.T., 2003. A\&A 412, 821.

Beuermann, K., Harrison, T.E., McArthur, B.E., Benedict, G.F., Gänsicke, B.T., 2004. A\&A 419, 291.

Bianchini, A., Skidmore, W., Bailey, J.M., Howell, S., Canterna, R., 2001. A\&A 367, 588.

Bilir, S., Karaali, S., Gilmore, G., 2006. MNRAS 366, 1295

Bilir, S., Cabrera-Lavers, A., Karaali, S., Ak, S., Yaz, E., Lopez-Corredoria, M., 2008. PASA 25, 69

Bonnet-Bidaud, J.M., Mouchet, M., Somova, T.A., Somov, N.N., 1996. A\&A 306, 199.

Bruch, A., Engel, A., 1994. A\&AS 104, 79.

Bruch, A., 2003. A\&A 409, 647.

Buser, R., Rong, J., Karaali, S., 1999. A\&A 348, 98.

Casares, J., Martinez-Pais, I.G., Marsh, T.R., Charles, P.A., Lazaro, C., 1996a. MNRAS 278, 219.

Casares, J., Mouchet, M., Martinez-Pais, I.G., Harlaftis, E.T., 1996b. MNRAS 282, 182.

Catalan, M.S., Schwope, A.D., Smith, R.C., 1999. MNRAS 310, 123.

Clemens, J.C., Reid, I.N., Gizis, J.E., O’Brien, M.S., 1998. ApJ 496, 352.

Cox, A.N., 2000. Allen's astrophysical quantities, New York: AIP Press;

Springer, Edited by Arthur N. Cox. ISBN: 0387987460.

de Kool, M., 1992. A\&A 261, 188.

de Kool, M., Ritter, H., 1993. A\&A 267, 397.

de Martino, D., Mouchet, M., Bonnet-Bidaud, J.M., Vio, R., Rosen, S.R.,

Mukai, K., Augusteijn, T., Garlick, M.A., 1995. A\&A 298849.

Dhillon, V.S., Jones, D.H.P., Marsh, T.R., 1994. MNRAS 266, 859.

Downes, R.A., Webbink, R.F., Shara, M.M., Ritter, H., Kolb, U., Duerbeck, H.W., 2001. PASP 113, 764.

Drew, J.E., Hoare, M.G., Woods, J.A., 1991. MNRAS 250, 144.

Duerbeck, H.W., 1999. IBVS No.4731.

Echevarria, J., de la Fuente, E., Costero, R., 2007. AJ 134, 262.

Fiedler, H., Barwig, H., Mantel, K.H., 1997. A\&A 327, 173.

Froning, C.S., Long, K.S., Baptista, R., 2003. AJ 126, 964.

Gänsicke, B.T., Dillon, M., Southworth, J., Thorstensen, J.R., Rodriguez-Gil, P., Aungwerojwit, A., Marsh, T.R., Szkody, P., Barros, S.C.C., Casares, 
J., de Martino, D., Groot, P.J., Hakala, P., Kolb, U., Littlefair, S.P., Martinez-Pais, I.G., Nelemans, G., Schreiber, M.R., 2009. MNRAS, in press (2009arXiv0905.3476G)

Girard, T.M., Dinescu, D.I., van Altena, W.F., Platais, I., Monet, D.G., Lopez, C.E., 2004. AJ 127, 3060.

Glenn, J., Howell, S.B., Schmidt, G.D., Liebert, J., Grauer, A.D., Wagner, R.M., 1994. ApJ 424, 967.

Greiner, J., Tovmassian, G.H., Di Stefano, R., Prestwich, A., GonzalezRiestra, R., Szentasko, L., Chavarra, C., 1999. A\&A 343, 183.

Greiner, J., Tovmassian, G., Orio, M., Lehmann, H., Chavushyan, V., Rau, A., Schwarz, R., Casalegno, R., Scholz, R.-D., 2001. A\&A 376, 1031.

Groot, P.J., Rutten, R.G.M., van Paradijs, J., 2001. A\&A 368, 183.

Hanson, R.B., Klemola, A.R., Jones, B.F., Monet, D.G., 2004. AJ 128, 1430.

Harlaftis, E.T., Marsh, T.R., 1996. A\&A 308, 97.

Harrison, T.E., Johnson, J.J., McArthur, B.E., Benedict, G.F., Szkody, P., Howell, S.B., Gelino, D.M., 2004. AJ 127, 460.

Harvey, D., Skillman, D.R., Patterson, J., Ringwald, F.A., 1995. PASP 107, 551.

Hellier, C., 2001. Cataclysmic Variable Stars, How and Why They Vary, Springer-Praxis Books in astronomy and space sciences, Cornwall, UK.

Hartley, L.E., Murray, J.R., Drew, J.E., Long, K.S., 2005. MNRAS 363, 285.

Hoard, D.W., Szkody, P., 1996. ApJ 470, 1052.

Hoard, D.W., Szkody, P., 1997. ApJ 481, 433.

Hoard, D.W., Szkody, P., Still, M.D., Smith, R.C., Buckley, D.A.H., 1998. MNRAS 294, 689.

Hoard, D.W., Thorstensen, J.R., Szkody, P., 2000. ApJ 537, 936.

Howell, S.B., Rappaport, S., Politano, M., 1997. MNRAS 287, 929.

Howell, S.B., Harrison, T.E., Campbell, R.K., Cordova, F.A., Szkody, P., 2006. AJ 131, 2216.

Huber, M.E., Howell, S.B., Ciardi, D.R., Fried, R., 1998. PASP 110, 784.

Johnson, D.R.H., Soderblom, D.R., 1987. AJ 93, 864.

Kafka, S., Tappert, C., Honeycutt, R.K., Bianchini, A., 2003. AJ 126, 1472.

Karaali, S., Bilir, S., Hamzaolu, E., 2004. MNRAS 355, 307.

Kharchenko, N. V., 2001. Kinematika Fizika Nebesnykh Tel, vol. 17, no. 5, p. 409.

King, A.R., 1988. QJRAS 29, 1.

King, A.R., Kolb, U., 1995. ApJ 439, 330.

King, A.R., Schenker, K., 2002. in The Physics of Cataclysmic Variables and Related Objects, ASP Conf. Ser., Vol.261, B.T. Gänsicke, K. Beuermann, K. Reinsch (eds.), 233.

Kislyuk, V., Yatsenko, A., Ivanov, G., Pakuliak, L., Sergeeva, T., 1999. Main Astronomical Observatory of National Academy of Science of Ukraine (VizieR On-line Data Catalog: I/261)

Klemola, A.R., Jones, B.F., Hanson, R.B., 1987. AJ 94, 501

Kolb, U., 1993. A\&A 271, 149. 
Kolb, U., Stehle, R., 1996. MNRAS 282, 1454.

Kolb, U., King, A.R., Ritter, H., 1998. MNRAS 298, 29.

Kolb, U., Baraffe, I., 1999. MNRAS 309, 1034.

Kolb, U., 2001. in The influence of binaries on stellar population studies, Astrophysics and Space Science Library (ASSL), Vol. 264, 321.

La Dous, C., 1991. A\&A 252, 100.

Liebert, J., Stockman, H.S., Williams, R.E., Tapia, S., Green, R.F., Rautenkranz, D., Ferguson, D.H., Szkody, P., 1982. ApJ 256, 594.

Littlefair, S.P., Dhillon, V.S., Marsh, T.R., Gänsicke, B.T., Southworth, J., Baraffe, I., Watson, C.A., Copperwheat, C., 2008. MNRAS 388, 1582.

Livio, M., Pringle, J.E., 1994. ApJ 427, 956.

Luyten, W.J., Hughes, H.S., 1965. A Search for faint blue stars. XXXVI. Proper Motions for Thirty-Eight Ancient Novae and U Geminorum Variables, (Minneapolis: Obs. Uni. Minnesota).

Marsh, T.R., 1988. MNRAS 231, 1117.

Marsh, T.R., 1999. MNRAS 304, 443.

Mason, E., Skidmore, W., Howell, S.B., Mennickent, R.E., 2001. ApJ 563, 351.

Matsumoto, K., Mennickent, R.E., Kato, T., 2000. A\&A 363, 1029.

Mauche, C.W., Raymond, J.C., Buckley, D.A.H., Mouchet, M., Bonnell, J., Sullivan, D.J., Bonnet-Bidaud, J., Bunk, W.H., 1994. ApJ 424, 347.

McArthur, B.E., Benedict, G.F., Lee, J., Lu, C.-L., van Altena, W.F. et al., 1999. ApJ 520, L59.

McArthur, B.E., Benedict, G.F., Lee, J., van Altena, W.F., Slesnick, C.L., et al., 2001. ApJ 560, 907.

Mennickent, R.E., Diaz, M., 1996. A\&A 309, 147.

Mennickent, R.E., Matsumoto, K., Arenas, J., 1999. A\&A 348, 466.

Mickaelian, A.M., Balayan, S.K., Ilovaisky, S.A., Chevalier, C., Veron-Cetty, M.-P., Veron, P., 2002. A\&A 381, 894.

Mihalas, D., Binney, J., 1981. in Galactic Astronomy, 2nd edition, Freeman, San Fransisco.

Morales-Rueda, L., Marsh, T.R., Steeghs, D., Unda-Sanzana, E., Wood, J.H., North, R.C., 2003. A\&A 405, 249.

Mukai, K., Bonnet-Bidaud, J.M., Charles, P.A., Corbet, R.H.D., Maraschi, L., Osborne, J.P., Smale, A.P., Treves, A., van der Klis, M., van Paradijs, J., 1986. MNRAS 221, 839.

Mukai, K., Charles, P.A., 1987. MNRAS 226, 209.

Naylor, T., Allan, A., Long, K.S., 2005. MNRAS 361, 1091.

Neustroev, V.V., 2002. A\&A 382, 974.

Neustroev, V.V., Zharikov, S., Michel, R., 2006. MNRAS 369, 369.

Nogami, D., Masuda, S., Kato, T., Hirata, R., 1999. PASJ 51, 115.

North, R.C., Marsh, T.R., Moran, C.K.J., Kolb, U., Smith, R.C., Stehle, R., 2000. MNRAS 313, 383.

North, R.C., Marsh, T.R., Kolb, U., Dhillon, V.S., Moran, C.K.J., 2002. MNRAS 337, 1215.

Paczynski, B., 1967. Acta Astron. 17, 287. 
Paczynski, B., Sienkiewicz, R., 1983. ApJ 268, 825.

Patterson, J., 1998. PASP 110, 1132.

Patterson, J., et al., 2003. PASP 115, 1308.

Patterson, J., Thorstensen, J.R., Vanmunster, T., Fried, R.E., Martin, B., Campbell, T., Robertson, J., Kemp, J., Messier, D., Armstrong, E., 2004. PASP 116, 516.

Peters, C.S., Thorstensen, J.R., 2005. PASP 117, 1386.

Peters, C.S., Thorstensen, J.R., 2006. PASP 118, 687.

Politano, M., 1996. ApJ 465, 338.

Potter, S.B., Romero-Colmenero, E., Watson, C.A., Buckley, D.A.H., Phillips, A., 2004. MNRAS 348, 316.

Pretorius, M.L., Knigge, C., O’Donoghue, D., Henry, J.P., Gioia, I.M., Mullis, C.R., 2007a. MNRAS 3821279.

Pretorius, M.L., Knigge, C., Kolb, U., 2007b. MNRAS 374, 1495.

Ramsay, G., Wheatley, P.J., 1998. MNRAS 301, 95.

Rappaport, S., Joss, P.C., Webbink, R.F., 1982. ApJ 254, 616.

Rappaport, S., Verbunt, F., Joss, P.C., 1983. ApJ 275, 713.

Reinsch, K., Beuermann, K., 1994. A\&A 282, 493.

Ringwald, F.A., 1994. MNRAS 270, 804.

Ringwald, F.A., Thorstensen, J.R., Hamwey, R.M., 1994. MNRAS 271, 323.

Ringwald, F.A., Thorstensen, J.R., Honeycutt, R.K., Smith, R.C., 1996a. AJ 111, 2077.

Ringwald, F.A., Thorstensen, J.R., Honeycutt, R.K., Robertson, J.W., 1996b. MNRAS 278, 125.

Ringwald, F.A., Chase, D.W., Reynolds, D.S., 2005. PASP 117, 1223.

Ritter, H., 2008. Mem. S.A.It. 1, 1.

Ritter, H., Burkert, A., 1986. A\&A 158, 161.

Ritter, H., Kolb, U., 2003. A\&A 404, 301.

Robin, A.C., Haywood, M., Créze, M., Ojha, D.K., Bienaymé, O., 1996. A\&A 305, 125.

Rodrigues, C.V., Cieslinski, D., Steiner, J.E., 1998. A\&A 335, 979.

Rodríguez-Gil, P., Gänsicke, B.T., Barwig, H., Hagen, H.-J., Engels, D., 2004. A\&A 424, 647.

Rodríguez-Gil, P., Gänsicke, B.T., Hagen, H.-J., Marsh, T.R., Harlaftis, E.T., Kitsionas, S., Engels, D., 2005. A\&A 431, 269.

Rodríguez-Gil, P., Gänsicke, B.T., Hagen, H.-J., Araujo-Betancor, S., Aungwerojwit, A., Allende Prieto, C., Boyd, D., Casares, J., Engels, D., Giannakis, O., Harlaftis, E.T., Kube, J., Lehto, H., Martínez-Pais, I.G., Schwarz, R., Skidmore, W., Staude, A., Torres, M.A.P., 2007a. MNRAS 377, 1747.

Rodríguez-Gil, Schmidtobreick, L., Gänsicke, B.T., 2007b. MNRAS 374, 1359.

Roelofs, G.H.A., Groot, P.J., Benedict, G.F., McArthur, B.E., Steeghs, D., Morales-Rueda, L., Marsh, T.R., Nelemans, G., 2007. ApJ 666, 1174.

Rolfe, D.J., Abbott, T.M.C., Haswell, C.A., 2002a. MNRAS 334, 699.

Rolfe, D.J., Abbott, T.M.C., Haswell, C.A., 2002b. ASP Conf. Ser. Vol. 261, 537. 
Rosen, S.R., Mittaz, J.P.D., Buckley, D.A., Layden, A.C., Clayton, K.L., McCain, C., Wynn, G.A., Sirk, M.M., Osborne, J.P., Watson, M.G., MNRAS 280, 1121.

Schenker, K., King, A.R., Kolb, U., Wynn, G.A., Zhang, Z., 2002. MNRAS 337, 1105.

Schlegel, E.M., Honeycutt, R.K., Kaitchuck, R.H., 1986. ApJ 307, 760.

Schmidt, G.D., Stockman, H.S., 2001, ApJ, 548, 410

Schwarz, R., Schwope, A.D., Beuermann, K., Burwitz, V., Fischer, J.-U., Fried, R., Lehmann, I., Mantel, K.-H., Mengel, S., Metzner, A., Misselt, K., Notni, P., Reinsch, K., Shafter, A., Thomas, H.-C., A\&A 338, 465.

Schwarz, R., Schwope, A.D., Staude, A., Remillard, R.A., 2005. A\&A 444, 213.

Schwarz, R., Schwope, A.D., Staude, A., Rau, A., Hasinger, G., Urrutia, T., Motch, C., 2007. A\&A 473, 511.

Schwope, A.D., Naundorf, C.E., Thomas, H.-C., Beuermann, K., 1991. A\&A $244,373$.

Schwope, A.D., Beuermann, K., Jordan, S., Thomas, H.-C., 1993. A\&A 278, 487.

Schwope, A.D., Mantel, K.-H., Horne, K., 1997. A\&A 319, 894.

Schwope, A.D., Catalan, M. S., Beuermann, K., Metzner, A., Smith, R.C., Steeghs, D., 2000. MNRAS 313, 533.

Schwope, A.D., Brunner, H., Buckley, D., Greiner, J., Heyden, K.V.D., Neizvestny, S., Potter, S., Schwarz, R., 2002. A\&A 396, 895.

Shahbaz, T., Wood, J.H., 1996. MNRAS 282, 362.

Sheets, H.A., Thorstensen, J.R., 2005. PASP, 117, 741.

Sheets, H.A., Thorstensen, J. R., Peters, C.J., Kapusta, A.B., Taylor, C. J., 2007. PASP 119, 494.

Skidmore, W., Mason, E., Howell, S.B., Ciardi, D.R., Littlefair, S., Dhillon, V.S., 2000. MNRAS 318, 429.

Skillman, D.R., Patterson, J., Thorstensen, J.R., 1995. PASP 107, 545.

Smak, J.I., 2002, Acta Astr. 52, 189.

Southworth, J., Gänsicke, B.T., Marsh, T.R., de Martino, D., Hakala, P., Littlefair, S., Rodrguez-Gil, P., Szkody, P., 2006. MNRAS 373, 687.

Southworth, J., Gänsicke, B.T., Marsh, T.R., 2008. proceedings of the 16th European White Dwarf Workshop (Barcelona, July 2008), in press. (2008arXiv0810.4823S)

Spruit, H.C., Ritter, H., 1983. A\&A 124, 267.

Staude, A., Schwope, A.D., Schwarz, R., 2001. A\&A 374, 588.

Steeghs, D., Marsh, T., Knigge, C., Maxted, P.F.L., Kuulkers, E., Skidmore, W., 2001. ApJ 562, L145.

Still, M.D., Marsh, T.R., Dhillon, V.S., Horne, K., 1994. MNRAS 267, 957.

Still, M.D., Dhillon, V.S., Jones, D.H.P., 1995. MNRAS 273, 863.

Still, M.D., Buckley, D.A.H., Garlick, M.A., 1998. MNRAS 299, 545.

Szkody, P., Desai, V., Hoard, D.W., 2000. AJ 119, 365.

Szkody, P., et al., 2002. AJ 123, 430. 
Szkody, P., et al., 2003. AJ 126, 1499.

Szkody, P., et al., 2004. AJ 128, 1882.

Szkody, P., Henden, A., Fraser, O.J., Silvestri, N.M., Schmidt, G.D., Bochanski, J.J., Wolfe, M.A., Agueros, M., Anderson, S.F., Mannikko, L., Downes, R.A., Schneider, D.P., Brinkmann, J., 2005. AJ 129, 2386.

Taam, R.E., Spruit, H.C., 2001. ApJ 561, 329.

Tappert, C., Wargau, W.F., Hanuschik, R.W., Vogt, N., 1997. A\&A 327, 231.

Tappert, C., Thorstensen, J.R., Fenton, W.H., Bennert, N., Schmidtobreick, L., Bianchini, A., 2001. A\&A 380, 533.

Taylor, C.J., Thorstensen, J.R., 1996. PASP 108, 894.

Thoroughgood, T.D., Dhillon, V.S., Watson, C.A., Buckley, D.A.H., Steeghs, D., Stevenson, M.J., 2004. MNRAS 353, 1135.

Thoroughgood, T.D., Dhillon, V.S., Steeghs, D., Watson, C.A., Buckley, D.A.H., Littlefair, S.P., Smith, D.A., Still, M., van der Heyden, K.J., Warner, B., 2005. MNRAS 357, 881.

Thorstensen, J.R., 1986. AJ 91, 940.

Thorstensen, J.R., 1997. PASP 109, 1241.

Thorstensen, J.R., Taylor, C.J., Becker, C.M., Remillard, R.A., 1997. PASP $109,477$.

Thorstensen, J.R., Taylor, C.J., 1997. PASP 109, 1359.

Thorstensen, J.R., Taylor, C.J., Kemp, J., 1998. PASP 110, 1405.

Thorstensen, J.R., 2000. PASP 112, 1269.

Thorstensen, J.R., Taylor, C.J., 2000. MNRAS 312, 629.

Thorstensen, J.R., Taylor, C.J., 2001. MNRAS 326, 1235.

Thorstensen, J.R., Fenton, W.H., 2002. PASP 114, 74.

Thorstensen, J.R., Fenton, W.H., Patterson, J., Kemp, J., Halpern, J., Baraffe, I., 2002a. PASP 114, 1117.

Thorstensen, J.R., Fenton, W.H., Patterson, J.O., Kemp, J., Krajci, T., Baraffe, I., 2002b. ApJ 567, L49.

Thorstensen, J.R., 2003. AJ 126, 3017.

Thorstensen, J.R., Fenton, W.H., 2003. PASP 115, 37.

Thorstensen, J.R., Fenton, W.H., Taylor, C.J., 2004. PASP 116, 300.

Thorstensen, J.R., Lépine, S., Shara, M., 2008. AJ 136, 2107.

Tovmassian, G.H., Greiner, J., Schwope, A.D., Szkody, P., Schmidt, G., Zickgraf, F.-J., Serrano, A., Krautter, J., Thiering, I., Zharikov, S.V., 2000. ApJ $537,927$.

Tovmassian, G.H., Zharikov, S.V., 2007. A\&A 468, 643

Unda-Sanzana, E., Marsh, T.R., Morales-Rueda, L., 2006. MNRAS 369, 805. Vande P.D., Smith, R.C., Hawkins, N.A., Martin, J.S., 2003. MNRAS 342, 151.

van der Heyden, K.J., Potter, S.B., Buckley, D.A.H., 2002. MNRAS 333, 241.

van Paradijs, J., Augusteijn, T., Stehle, R., 1996. A\&A 312, 93.

Vaytet, N.M.H., O’Brien, T.J., Rushton, A.P., 2007. MNRAS 380, 175.

Verbunt, F., Zwaan, C., 1981. A\&A 100, L7.

Wagner, R.M., Thorstensen, J.R., Honeycutt, R.K., Howell, S.B., Kaitchuck, 
R.H., Kreidl, T.J., Robertson, J.W., Sion, E.M., Starrfield, S.G., 1998. AJ $115,787$.

Warner, B., 1995. Cataclysmic Variable Stars, Cambridge Univ. Press, Cambridge.

Watson, M.G., Rosen, S.R., O'Donoghue, D., Buckley, D.A.H., Warner, B., Hellier, C., Ramseyer, T., Done, C., Madejski, G., 1995. MNRAS 273, 681.

Watson, C.A., Dhillon, V.S., Rutten, R.G.M., Schwope, A.D., 2003. MNRAS 341, 129.

Webbink, R.F., Wickramasinghe, D.T., 2002. MNRAS 335, 1.

Weight, A., Evans, A., Naylor, T., Wood, J.H., Bode, M.F., 1994. MNRAS 266, 761 .

Welsh, W.F., Horne, K., Gomer, R., 1995. MNRAS 275, 649.

Wielen, R., 1977. A\&A 60, 263.

Wielen, R., Dettbarn, C., Fuchs, B., Jahreiss, H., Radons, G., 1992. in The Stellar Populations of Galaxies, IAU Symp. 149, B. Barbuy, A. Renzini (eds.), 81.

Willems, B., Kolb, U., Sandquist, E.L., Taam, R.E., Dubus, G., 2005. ApJ 635, 1263.

Willems, B., Taam, R.E., Kolb, U., Dubus, G., Sandquist, E.L., 2007. ApJ $657,465$.

Witham, A.R., Knigge, C., Aungwerojwit, A., Drew, J.E., Gänsicke, B.T., Greimel, R., Groot, P.J., Roelofs, G.H.A., Steeghs, D., Woudt, P.A., 2007. MNRAS 382, 1158.

Wolf, S., Barwig, H., Bobinger, A., Mantel, K.-H., Simic, D., 1998. A\&A 332, 984.

Wu, K., Wickramasinghe, D.T., 1993. MNRAS 265, 115.

Wu, X., Li, Z., Gao, W., Leung, K.-C., 2001. ApJ 549, L81.

Zacharias, N., Monet, D.G., Levine, S.E., Urban, S.E., Gaume, R., Wycoff, G.L., 2005. VizieR On-line Data Catalog: I/297.

Zharikov, S. V., Tovmassian, G. H., Napiwotzki, R., Michel, R., Neustroev, V., 2006. A\&A 449, 645. 